\title{
Decomposition and Reconstruction of multidimensional signals by radial functions with tension parameters
}

\author{
Mira Bozzini · Christophe Rabut • \\ Milvia Rossini
}

Received: date / Accepted: date

\begin{abstract}
The aim of the paper is to construct a multiresolution analysis of $L^{2}\left(\mathbb{R}^{d}\right)$ based on generalized kernels which are fundamental solutions of differential operators of the form $\prod_{\ell=1}^{m}\left(-\Delta+\kappa_{\ell}^{2} I\right)$. We study its properties and provide a set of pre-wavelets associated with it, as well as the filters which are indispensable to perform decomposition and reconstruction of a given signal, being very useful in applied problems thanks to the presence of the tension parameters $\kappa_{\ell}$.
\end{abstract}

Keywords Generalized Whittle-Matérn kernels · radial basis functions · multiresolution analysis $\cdot$ wavelets $\cdot$ filters $\cdot$ tension parameters

Mathematics Subject Classification (2000) 41A05 - 41063 - 41065 . $65 \mathrm{D} 05 \cdot 65 \mathrm{D} 15$

\section{Introduction}

In [5], the fundamental solutions associated with the differential operator defined for a given integer $m>d / 2$ and $m$ non negative real numbers $\left\{\kappa_{\ell}\right\}_{\ell=1}^{m}$

\author{
Mira Bozzini \\ Dipartimento di Matematica e Applicazioni, Università di Milano-Bicocca, via Roberto \\ Cozzi 55, 20125 Milano, Italy \\ E-mail: mira.bozzini@unimib.it \\ Christophe Rabut \\ INSA Toulouse (Institut National des Sciences Appliques), 135, Avenue de Rangueil, 31077 \\ Toulouse Cedex 4 France \\ E-mail: christophe.rabut@insa-toulouse.fr \\ Milvia Rossini \\ Dipartimento di Matematica e Applicazioni, Università di Milano-Bicocca, via Roberto \\ Cozzi 55, 20125 Milano, Italy \\ E-mail: milvia.rossini@unimib.it
}


by

$$
L=\prod_{\ell=1}^{m}\left(-\Delta+\kappa_{\ell}^{2} I\right), \quad m>\frac{d}{2}
$$

were studied. The parameters $\left\{\kappa_{\ell}\right\}_{\ell=1}^{m}$ can be considered as tension parameters.

Such operators determine a new family of radial functions. The choice $\kappa_{\ell}>0, \ell=1, \ldots m$, provides strictly positive definite functions the so called generalized Whittle-Matérn kernels which have a computable analytic expression. Suitable choices of the parameter $\kappa_{\ell}$ give known classes of radial kernels. For $\kappa_{\ell}=0, \ell=1, \ldots m$, we get the (conditionally positive definite) polyharmonic splines, while if all the parameters are equal to $\kappa>0$ we get a scaled Whittle-Matérn-Sobolev radial kernel which is positive definite. When $n<m$ values of the $\kappa_{\ell}$ are zero, the resulting functions are are linear combinations of generalized Whittle-Matérn kernels with polyharmonic functions.

In this paper we discuss the properties of this new family of functions that offers the opportunity of choosing an element of the family according to the applied problem to deal with. That is, strictly positive definite functions or conditionally positive definite functions of order $2 n-1<m$ that are fundamental solutions of known differential operators. Connecting (conditionally) positive definite kernels to fundamental functions, provides an interpretation of native spaces as generalized Sobolev spaces associated to $L$ (see [10]). Here, we mainly consider the case when the parameters $\kappa_{\ell}$ are all positive. In this case we denote by $u$ the fundamental solution of $L$.

The Generalized Whittle-Matérn function (GWM) $u$ generates a (non separable) Non-Stationary Multiresolution Analysis (NSMRA) of $L^{2}\left(\mathbb{R}^{d}\right)$ as happens for other radial basis functions such as Gaussians and multiquadrics (see e.g. [7]), [18]). In addition it is possible to construct the filters that allow a fast decomposition and reconstruction of a multidimensional signal. In this paper, we provide all technical and theoretical tools to manipulate them.

One of the interests of this new NSMRA, compared for instance to the stationary one generated by polyharmonic B-splines (see e.g. [2], [16]), is its flexibility given by the presence of the parameters $\kappa_{\ell}$. The choice of $\kappa_{\ell}$ depends on the applications, small values provide smooth functions, while large values provide peaked functions useful to capture specific features. Some applications need for instance to approximate quantities with functions (or data) whose irregularities are between two orders of irregularities. This is the case for example, in medical imaging, for Magnetic Resonance Images, whose rate of decay in the Fourier domain is somewhere between two integer numbers. Fractional polyharmonic splines [20] are an answer for that, and the functions studied here, linear combinations of GWM kernels, are another answer which deserves to be studied and developed. Indeed they are between two wellknown function families and their advantages are illustrated in the example below. Their interest is the possibility for the user to have tension parameters. In order to show the effects of the parameters $\left\{\kappa_{\ell}\right\}_{\ell=1}^{m}$, and for a better visibility, we give here an examples for univariate interpolation. Of course their impact is similar in higher dimensions. Let us consider the classical Akima's 
data set [1] and select $m=2$. Fig.1.1 shows the different behaviours of the GWM interpolants with a fixed value of $\kappa_{1}$ and increasing values of $\kappa_{2}$. When $\kappa_{2}$ increases, the interpolant preserves the shape of the data. In Fig. 1.2, we compare the results provided by the GWM kernel $u$ with those obtained by Polyharmonic Spline $\|x\|^{2 m-1}$ (PS) (which in one dimension is an odd degree polynomial spline) and the scaled Whittle Matérn kernel (WM) $S_{m, 1, \kappa}(3.4)$ for some choice of the scale parameter $\kappa$.
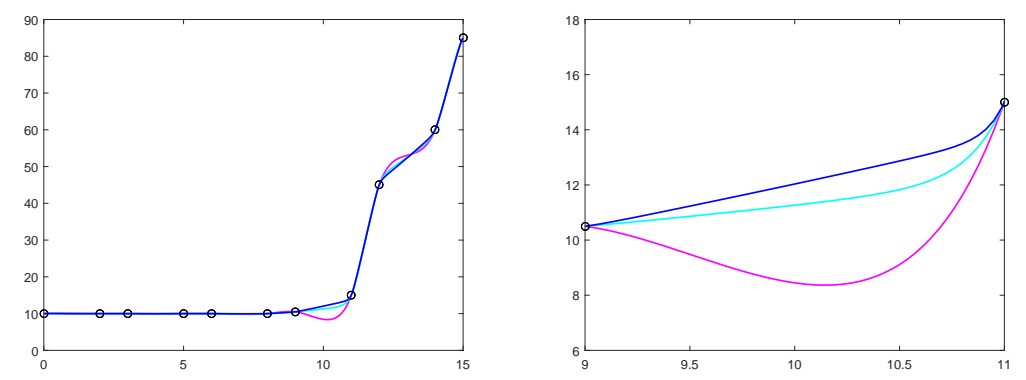

Fig. 1.1 GWM-interpolants with $\kappa_{1}=0.1$. Magenta line: $\kappa_{2}=2$. Ciano line: $\kappa_{2}=6$ Blue-line: $\kappa_{2}=12$. Right: The interpolants on the interval $[9,11]$.
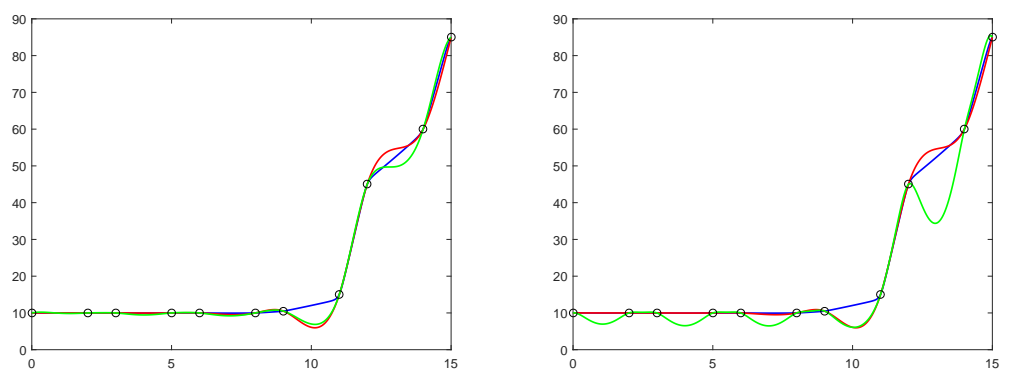

Fig. 1.2 Blue line: GWM-interpolant with $\kappa_{1}=0.1$, and $\kappa_{2}=12$. Red line: PS-interpolant. Green line: (left) WM-interpolant with $\kappa=1$, (right) WM-interpolant with $\kappa=2$.

Furthermore these functions support a NSMRA, which is of interest, and the study of the properties of this NSMRA is of course important for their use, and is the main aim of this paper. In Section 6.2 the reader will see the crucial role played by the parameters $\left\{\kappa_{\ell}\right\}_{\ell=1}^{m}$ also in detecting important features of a signal. These examples highlight the details in a decomposition process and so show that these functions may be useful for edge detection.

The paper is organized as follows. In Section 2.1 we give some notations and definitions. In Section 2.2 we present the already known polyharmonic and Whittle-Matérn radial kernels. In Section 3 we discuss their generalization and 
study their key properties such us the minimization property. Section 4.1 is devoted to the construction of a NSMRA based on the GWM function $u$, and since $u$ is a non-separable function, we consider a generic scaling matrix $M$, but we require that $M$ is a similarity, i.e. i.e a matrix of the form $M=$ $\rho M_{0}$, where $M_{0}$ is an orthogonal matrix, and $\rho$ is a real number such that $|\operatorname{det} M|=\left|\rho^{d}\right|$ is an integer $\geq 2$. In this way the lattices generated by $M$ and $M^{t}$ are the same and the lattices $M^{j} \mathbb{Z}^{d}, j \in \mathbb{Z}$ scale uniformly in every direction, important property in multiresolution settings. In order to have a function satisfying a non-stationary refinement equation, we introduce in Section 4.1.1 the cardinal function associated with $u$. The construction of a set of pre-wavelets is presented in Section 4.2. It makes use of the differential operator $L^{2}$. We remark that this strategy is often used when dealing with scaling functions which are connected with fundamental solutions of some differential operator (see e.g [2], [16]). The more general setting of operatorlike wavelets is presented in [12]. Unlike [12], we also provide in Section 5 the reconstruction and decomposition filters, and give in Section 6 a short discussion on how to decompose/reconstruct a signal via convolutions, and we provide some numerical examples of signal decomposition that visualize the effectiveness of the proposed NSMRA.

\section{Preliminaries}

\subsection{Basic notations and definitions}

Throughout this paper, $d$ is the dimension of the space and $m$ is an integer such that $m>d / 2$. We use the multi-index notation, in particular for $s \in \mathbb{Z}^{d}$, $|s|=\sum_{i=1}^{d}\left|s_{i}\right|$. A function $f$ is from $\mathbb{R}^{d}$ to $\mathbb{R}$ and a vector $v$ has indices in $\mathbb{Z}^{d}$ and its components belong to $\mathbb{R}$.

The symbol $*$ denotes the convolution operator between functions $f$ and $g$ in $L^{1}\left(\mathbb{R}^{d}\right)$, between two vectors $v$ and $\xi$ in $l^{1}\left(\mathbb{Z}^{d}\right)$, or between a vector $v$ in $l^{1}\left(\mathbb{Z}^{d}\right)$ and a function $f$ in $L^{1}\left(\mathbb{R}^{d}\right)$, i.e. :

$$
f * g:=\int_{\mathbb{R}^{d}} f(x) g(\cdot-x) d x, \quad v * \xi:=\sum_{s \in \mathbb{Z}^{d}} v_{s} \xi \cdot-s, \quad v * f:=\sum_{s \in \mathbb{Z}^{d}} v_{s} f(\cdot-s) .
$$

We denote by $\|\cdot\|$ the usual euclidean norm on $\ell^{2}\left(\mathbb{Z}^{d}\right)$ or on $L^{2}\left(\mathbb{R}^{d}\right)$. We use standard notation for the inner product on $L^{2}\left(\mathbb{R}^{d}\right)$, and on $\mathbb{R}^{d}$ i.e.

$$
x \cdot y=\sum_{\ell=0}^{d} x_{\ell} y_{\ell}, \quad \text { and } \quad(f, g):=\int_{\mathbb{R}^{d}} f(x) g(x) d x .
$$

We use ${ }^{\wedge}$ for the Fourier transform, i.e. for any function $f$ in $L^{1}\left(\mathbb{R}^{d}\right)$ and any vector $\xi \in \ell^{1}\left(\mathbb{Z}^{d}\right)$,

$$
\widehat{f}(\omega):=\int_{\mathbb{R}^{d}} f(x) e^{-i \omega \cdot x} d x, \quad \widehat{\xi}(\omega):=\sum_{k \in \mathbb{Z}^{d}} \xi_{k} e^{-i k \cdot \omega}, \quad \omega \in \mathbb{R}^{d} .
$$


Thus $\xi$ is the vector of the Fourier coefficients of $\widehat{\xi}$. The map $\widehat{\imath} \xi \rightarrow \widehat{\xi}$ is linear and continuous (of norm 1 ) from $l^{1}\left(\mathbb{Z}^{d}\right)$ into $C\left(\mathbb{T}^{d}\right)$ (continuous functions over the $d$-dimensional torus $\mathbb{T}^{d}=[-\pi, \pi)^{d}$. We also use ${ }^{-}$for the Fourier transform of distributions.

For any natural number $n$, let $D^{n} f$ denotes the vector of all the $d^{n}$ order $n$ derivatives of the function $f$ (note that, in some circumstances, the derivatives must be taken in the sense of distributions). The Laplacean operator $\Delta$ is $\sum_{\ell=1}^{d} \frac{\partial^{2}}{\partial x_{\ell}^{2}}$ and Dirac denotes the usual Dirac distribution.

We define $H^{m}\left(\mathbb{R}^{d}\right)$ to be the space of functions on $\mathbb{R}^{d}$ whose all derivatives up to order $m$ are in $L^{2}\left(\mathbb{R}^{d}\right)$, endowed with the usual scalar product

$$
(f, g)_{m}=\int_{\mathbb{R}^{d}} \sum_{n=0}^{m}\left(D^{n} f\right)(x) \cdot\left(D^{n} g\right)(x) d x
$$

and the associated norm

$$
\|f\|_{m}=\left(\int_{\mathbb{R}^{d}} \sum_{n=0}^{m}\left\|\left(D^{n} f\right)(x)\right\|^{2} d x\right)^{\frac{1}{2}} .
$$

As it is known (from Krylov theorem), since $m>d / 2$, the elements of $H^{m}\left(\mathbb{R}^{d}\right)$ are actually continuous functions and $H^{m}\left(\mathbb{R}^{d}\right)$ endowed with this norm is a Hilbert space.

We say that a linear transformation $M$ on $\mathbb{R}^{d}$ is an acceptable dilation for $\mathbb{Z}^{d}$ (see e.g. [14]) if $M \mathbb{Z}^{d} \subset \mathbb{Z}^{d}$, and all the eigenvalues of $M$ have modulus greater than one. These properties imply that $|\operatorname{det} M|$ is an integer $\geq 2$.

Important acceptable dilations for $Z^{d}$ are similarities, i.e. matrices of the form $M=\rho M_{0}$, where $M_{0}$ is an orthogonal matrix, and $\rho$ is a real number such that $|\operatorname{det} M|=\left|\rho^{d}\right|$ is an integer $\geq 2$. In the case $d=2$, these matrices are all of the form

$$
\left(\begin{array}{cc}
q & p \\
-p & q
\end{array}\right) \quad \text { or } \quad\left(\begin{array}{cc}
q & p \\
p & -q
\end{array}\right)
$$

with $q, p \in \mathbb{Z}$. They include the classic dyadic matrix $2 I$ and the quincunx matrix $(q=p=1)$ which gives $|\operatorname{det} M|=2$. When using a similarity, the lattices generated by $M$ and $M^{t}$ are the same and the lattices $M^{j} \mathbb{Z}^{d} j \in \mathbb{Z}$ scale uniformly in every direction.

If $M$ is an acceptable dilation of $\mathbb{Z}^{d}$ then a coset of $M \mathbb{Z}^{d}$ is a set of the form

$$
e+M \mathbb{Z}^{d}=\left\{e+M k: k \in \mathbb{Z}^{d}\right\},
$$

where $e$ is any element of $\mathbb{Z}^{d}$ which is referred to as a representative of the coset. Any pair of cosets is either identical or disjoint so that the collection of all cosets (denoted by $\mathbb{Z}^{d} / M \mathbb{Z}^{d}$ ) consists of disjoint cosets whose union is $\mathbb{Z}^{d}$. The number of disjoint cosets is $|\operatorname{det} M|$. A subset $\mathcal{E}$ of $\mathbb{Z}^{d}$ is said to be a full 
collection of representatives of $\mathbb{Z}^{d} / M \mathbb{Z}^{d}$ if it contains exactly $|\operatorname{det} M|$ elements and

$$
\bigcup_{e \in \mathcal{E}}\left(e+M \mathbb{Z}^{d}\right)=\mathbb{Z}^{d}
$$

This means that each $\ell \in \mathbb{Z}^{d}$ can be uniquely written as

$$
\ell=e+M k \quad \text { for some } e \in \mathcal{E} \text {, and } k \in \mathbb{Z}^{d} .
$$

The collection of representative $\mathcal{E}$ is $\mathcal{E}:=M\{0,1\}^{d} \cap \mathbb{Z}^{d}$. We denote by $\mathcal{E}_{0}$ the set $\mathcal{E} \backslash\{0\}^{d}$.

The matrix $M$ defines a sequence of lattices $\left\{M^{j} \mathbb{Z}^{d}, j \in \mathbb{Z}\right\}$ that can be decomposed as

$$
\bigcup_{e \in \mathcal{E}}\left(M^{j} e+M^{j+1} \mathbb{Z}^{d}\right)=M^{j} \mathbb{Z}^{d} .
$$

Let $N=M^{t}$ be the transposed matrix of $M$; the lattice $2 \pi N^{-j} \mathbb{Z}^{d}$ is called the lattice dual to $M^{j} \mathbb{Z}^{d}$ and the role played by the torus $\mathbb{T}^{d}$ (see e.g. [15]) is now played by the well-defined and unique tiling cell: the Voronoi cell $\Omega_{j}$ that is the set of points of $\mathbb{R}^{d}$ closer to zero than to any other point of the lattice $2 \pi N^{-j} \mathbb{Z}^{d}$

$$
\Omega_{j}=\left\{\omega \in \mathbb{R}^{d}:\|\omega\| \leq\left\|\omega-2 \pi N^{-j} l\right\| \text { for all } l \in \mathbb{Z}^{d} \backslash\{0\}\right\} .
$$

$\Omega_{j}$ is also referred to as fundamental region of the lattice $2 \pi N^{-j} \mathbb{Z}^{d} . \Omega_{j}$ is a bounded measurable set with $\left|\Omega_{j}\right|=2 \pi|\operatorname{det}(M)|^{-j}$, and

$$
\left.\Omega_{j} \bigcap\left(\Omega_{j}+2 \pi N^{-j} k\right)=\emptyset\right\} k \neq 0 \text { and } \mathbb{R}^{d}=\bigcup_{k \in \mathbb{Z}^{d}}\left(\Omega_{j}+M \pi N^{-j} k\right) .
$$

For a function $g \in L^{1}\left(\mathbb{R}^{d}\right)$ such that its Fourier transform $\widehat{g}$ is absolutely summable, the variant of the standard Poisson summation formula is

$$
\sum_{k \in \mathbb{Z}^{d}} g\left(M^{-j} k\right) e^{-i\left(M^{-j} k, \omega\right)}=|\operatorname{det} M|^{j} \sum_{k \in \mathbb{Z}^{d}} \widehat{g}\left(\omega-2 \pi N^{j} k\right), \quad \omega \in \Omega_{j},
$$

and the convergence of the sums is in $L^{1}\left(\Omega_{j}\right)$. If $g$ satisfies the two estimates $|g(x)|=O((1+\|x\|))^{-d-\epsilon}$ and $|\widehat{g}(\omega)|=O((1+\|\omega\|))^{-d-\epsilon}$, for positive $\epsilon$, the above identity holds pointwise.

Definition 2.1 A sequence $\left\{V_{j}\right\}_{j \in \mathbb{Z}}$ of closed linear subspaces of $L^{2}\left(\mathbb{R}^{d}\right)$ is called non-stationary multiresolution analysis associated with $\left(\mathbb{Z}^{d}, M\right)$ if

1. $\mathcal{V}_{j} \subset \mathcal{V}_{j+1}$,

2. $\bigcup_{j \in \mathbb{Z}} \mathcal{V}_{j}=L^{2}\left(\mathbb{R}^{d}\right)$,

3. $\bigcap_{j \in \mathbb{Z}} \mathcal{V}_{j}$ is at most one dimensional,

4. each $\mathcal{V}_{j}$ is shift invariant relative to the lattice $M^{-j} \mathbb{Z}^{d}$

$$
f \in \mathcal{V}_{j} \Rightarrow f\left(\cdot-M^{-j} k\right) \in \mathcal{V}_{j}, \quad \text { for all } k \in \mathbb{Z}^{d},
$$


5. for each $j$, there is an element $\varphi_{j} \in \mathcal{V}_{j}$ such that $\left\{\varphi_{j}\left(\cdot-2^{-j} k\right), k \in \mathbb{Z}^{d}\right\}$ is a Riesz basis of $\mathcal{V}_{j}$.

Note that this definition is a general one, in order to take into account the special case when the dimension of $\bigcap_{j \in \mathbb{Z}} \mathcal{V}_{j}$ is one. Actually this may happen (see [8]) for instance when $\varphi_{j}=\varphi$ for each $j$ (not to be confused with the stationary case), since in this case $\varphi \in \bigcap_{j \in \mathbb{Z}} \mathcal{V}_{j}$.

The shifts $\left\{\varphi\left(\cdot-M^{-j} k\right)\right\}_{k \in \mathbb{Z}^{d}}$ form a Riesz basis for $\mathcal{V}_{j}$ if there are positive constants $0<\mathcal{A}_{j} \leq \mathcal{B}_{j}<\infty$, such that for any vector $v^{j} \in \ell^{2}\left(\mathbb{Z}^{d}\right)$

$$
\mathcal{A}_{j}\left\|v^{j}\right\|^{2} \leq \int_{\mathbb{R}^{d}}\left|\sum_{k \in \mathbb{Z}^{d}} v_{k}^{j} \varphi\left(x-M^{-j} k\right)\right|^{2} d x \leq \mathcal{B}_{j}\left\|v^{j}\right\|^{2} .
$$

This condition is equivalent to

$$
\mathcal{A}_{j} \leq \sum_{k \in \mathbb{Z}^{d}}\left|\widehat{\varphi}\left(\omega+2 \pi N^{j} k\right)\right|^{2} \leq \mathcal{B}_{j}, \quad \omega \in \mathbb{R}^{d} .
$$

\subsection{Polyharmonic and Whittle-Matérn-Sobolev kernels}

Connections between either splines and Green's functions or radial basis functions and Green's functions have repeatedly been used and made over the past decades (see e.g. [17], [19], [10], and the references therein).

A very special case are Polyharmonic splines introduced in [9] that have been well studied by several authors because of their interesting properties (see e.g. [6], [11], [16]).

Let $m \in \mathbb{N}$ be such that $2 m-d>0$, and let $v_{2 m-d}$ be a fundamental solution of the elliptic equation of the form $-\Delta^{m} u=0$ on $\mathbb{R}^{d}$. We have

$$
v_{2 m-d}(x)= \begin{cases}C_{m, d}\|x\|^{2 m-d} & \text { if } 2 m-d \notin 2 \mathbb{Z} \\ C_{m, d}\|x\|^{2 m-d} \log \|x\| & \text { if } 2 m-d \in 2 \mathbb{Z} .\end{cases}
$$

The constant $C_{m, d}$ is such that their generalized Fourier transform is $\widehat{v}_{2 m-d}(\omega)=$ $(-1)^{m}\|\omega\|^{-2 m}$. A $m$-harmonic spline is in the form $\sum_{i \in I} \lambda_{i} v_{2 m-d}\left(\cdot-x_{i}\right)+$ $p_{m-1}$ where the $\left\{x_{i}\right\}_{i \in I}$ are (finitely or infinitely many) discrete points in $\mathbb{R}^{d}$, the $\lambda_{i}$ are associated real numbers meeting the relation $\sum_{i \in I} \lambda_{i} q\left(x_{i}\right)=0$ for any polynomial $q$ of degree at most $m-1$, and $p_{m-1}$ is a polynomial of degree at most $m-1$.

We remind the reader that $m>d / 2$ implies that $m$-harmonic splines are conditionally positive definite functions of orders $\lceil m-d / 2\rceil$ if $m-d / 2 \notin \mathbb{Z}$ and $1+m-d / 2$ if $m-d / 2 \in \mathbb{N}_{+}$.

Another prominent case is the positive definite Whittle-Matérn-Sobolev function $S_{m, d}$ defined by

$$
S_{m, d}(x)=\|x\|^{m-d / 2} K_{m-d / 2}(\|x\|), x \in \mathbb{R}^{d}
$$


involving the Bessel function $K_{\nu}$ of the third kind. Its Fourier transform is (up to positive factors) $\left(\|\cdot\|^{2}+1\right)^{-m}$, and it is a fundamental solution of the elliptic differential operators $(-\Delta+I)^{m}$.

\section{The generalized Whittle-Matérn-Sobolev functions}

Both class of function were generalized in [5] by considering fundamental solutions of more general elliptic operators of the form

$$
L:=\prod_{\ell=1}^{m}\left(-\Delta+\kappa_{\ell}^{2} I\right), \quad m>\frac{d}{2} .
$$

When $\kappa_{\ell}^{2}, 1 \leq \ell \leq m$ are positive real numbers these differential operators have positive radial Fourier transforms

$$
\widehat{L}=\prod_{\ell=1}^{m}\left(\|\cdot\|^{2}+\kappa_{\ell}^{2}\right)
$$

within bounds of the form

$$
0<\prod_{j=1}^{m} \kappa_{j}^{2} \leq \widehat{L}(\omega) \leq C\left(\|\omega\|^{2}+1\right)^{m} \text { for all } \omega \in \mathbb{R}^{d} .
$$

We denote by $u$ a fundamental solution of the operator $L$ defined in (3.1), i.e. a function meeting $L u=$ Dirac. All functions $u$ are inverse Fourier transforms of $\widehat{u}$ defined for any $\omega \in \mathbb{R}^{d}$ by

$$
\widehat{u}(\omega)=\left(\prod_{\ell=1}^{m}\left(\|\omega\|^{2}+\kappa_{\ell}^{2}\right)\right)^{-1},
$$

and they exist classically as continuous radial kernels. If all $\kappa_{\ell}$ are positive and equal, we have to find the inverse Fourier transform of $\left(\|\omega\|^{2}+\kappa^{2}\right)^{-m}$, and by standard rules of Fourier transforms, the result is the scaled WhittleMatérn-Sobolev radial function defined for any $m>d / 2, \kappa>0$ and $x \in \mathbb{R}^{d}$ by

$$
S_{m, d, \kappa}(x)=\frac{2^{1-m}}{(m-1) !}\left(\frac{\|x\|}{\kappa}\right)^{m-d / 2} K_{m-d / 2}(\kappa\|x\|) .
$$

For strictly positive values of the $\kappa_{\ell}$, it was proved in [5] that $u$, the Generalized Whittle-Matérn-Sobolev kernel, can be written explicitly as a divided difference of order $m$ of $S_{1, d, \sqrt{2 z}}$ with respect to $z\left([\cdot, \cdot, \ldots, \cdot]_{z} S_{1, d, \sqrt{2 z}}\right)$. Namely the inverse $d$-variate Fourier transform of (3.3) for arbitrary positive numbers $\kappa_{1}, \ldots, \kappa_{m}$ is

$$
u=2^{-m+1}(-1)^{m-1}\left[\kappa_{1}^{2} / 2, \ldots, \kappa_{m}^{2} / 2\right]_{z}\left(\frac{\|\cdot\|}{\sqrt{2 z}}\right)^{1-d / 2} K_{1-d / 2}(\|\cdot\| \sqrt{2 z}) .
$$



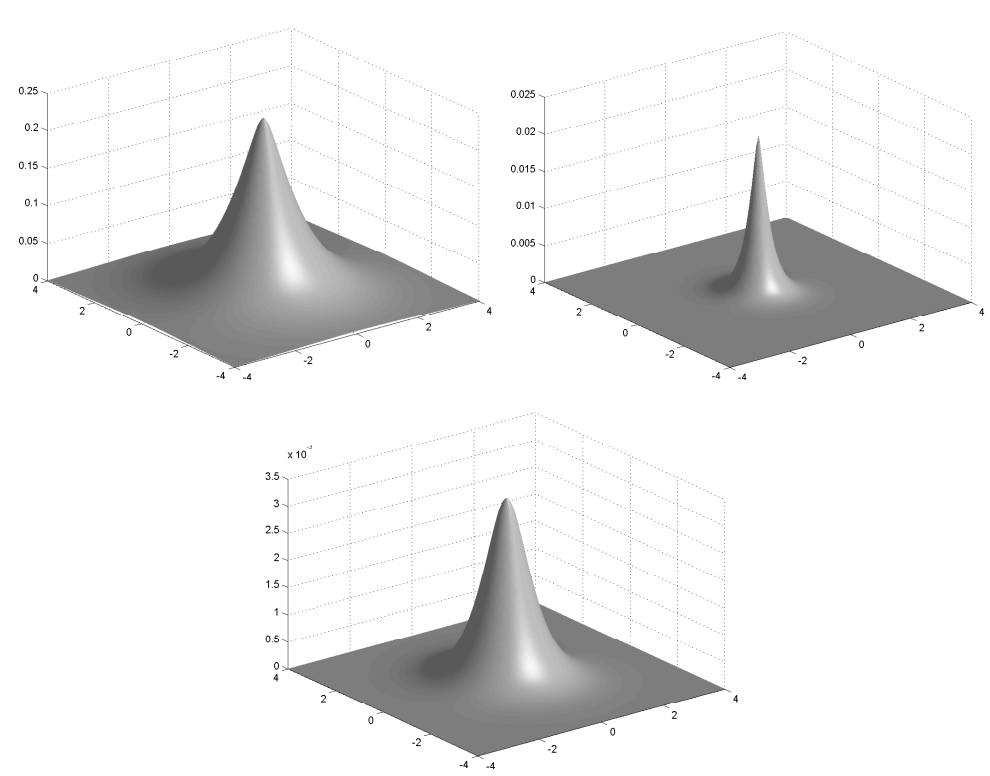

Fig. 3.1 Generalized Whittle-Matérn-Sobolev kernel $u$. Top left: $m=2, \kappa_{1}=1, \kappa_{2}=2$. Top right: $m=2, \kappa_{1}=3, \kappa_{2}=7$. Bottom: $m=3, \kappa_{1}=2, \kappa_{2}=3, \kappa_{3}=4$.

In Figure 3.1, we show the graphs of $u$ for $d=2, m=2$ and $m=3$.

Suppose that all $\kappa_{\ell}$ are nonzero, then $\widehat{u}$ is strictly positive and so $u$ is a positive definite function. As a consequence the native space of $u$ does not include the degree one polynomials. Suppose on the opposite that $n$ coefficients $\kappa_{\ell}, \ell=m-n+1, \ldots, m$ are equal to zero, and let's call $w$ the fundamental solution of $L$, then the Fourier transform (3.3) gets singularities at the origin and meets

$$
\widehat{w}(\omega)=\|\omega\|^{-2 n}\left(\prod_{\ell=1}^{m-n}\left(\|\omega\|^{2}+\kappa_{\ell}^{2}\right)\right)^{-1}, \quad \omega \in \mathbb{R}^{d} .
$$

Since it is a nonnegative function, and $\omega=0$ is the unique singularity of order $2 n, w$ is a conditionally positive function of order $2 n-1$. The resulting functions $w$ (see [5]) are linear combinations of GWM kernels with polyharmonic functions.

In this case $\Delta^{n}$ is a factor of $L$, and any $v$ in the form $v=w+p_{2 n-1}$ (where $p_{2 n-1}$ is a polynomial of degree at most $2 n-1$ ) is also a fundamental solution of $L$. As a consequence of it, the associated native space includes the degree $2 n-1$ polynomials.

The differential operator $L$ can also be seen as a multivariate extension of the one dimensional operator considered e.g. in [13], [4] and [19]: for particular choices of the parameters this operator is $D^{2}\left(D^{2}-\kappa^{2} I\right)$, which in our case becomes $-\Delta\left(-\Delta+\kappa^{2} I\right)$. 
In the rest of the paper we focus on the case in which all the parameters $\kappa_{\ell}$ are positive, and on the corresponding function $u$.

\subsection{Further properties of the GWM kernel $u$}

For $d \geq 2$, the kernels involved in the divided differences have singularities at zero. But the divided differences cancel these singularities, and then the resulting kernel is well-defined at zero and $2 m-d$ provides the class of regularity. If $2 m-d \geq 1, u \in C^{2 m-1-d}\left(\mathbb{R}^{d}\right)$.

The function $u$ decays exponentially at infinity, actually it is a linear combination of kernels involving the Bessel functions $K_{1-d / 2}\left(\kappa_{\ell}\|x\|\right), \ell=1, \ldots, m$ which, for large values of $\|x\|$, behave as $\exp \left(-\kappa_{\ell}\|x\|\right)$.

Let the positive numbers $\alpha_{\ell}$ be such that $p_{m}(x)=\prod_{\ell=1}^{m}\left(x+\kappa_{\ell}^{2}\right):=$ $\sum_{\ell=0}^{m} \alpha_{\ell} x^{\ell}$. We simply have $\alpha_{0}=\prod_{\ell=1}^{m} \kappa_{\ell}, \alpha_{1}=\sum_{\ell=1}^{m} \prod_{s \neq \ell} \kappa_{s}, \ldots, \alpha_{m-1}=$ $\sum_{\ell=1}^{m} \kappa_{\ell}, \alpha_{m}=1$, more generally, $\alpha_{\ell}$ is the sum of all products of $m-\ell$ different $\kappa_{\ell}$ and we can write

$$
L=p_{m}(-\Delta)=\sum_{\ell=0}^{m} \alpha_{\ell}(-\Delta)^{\ell}
$$

and

$$
\widehat{L}(\omega)=p_{m}\left(\|\omega\|^{2}\right)=\sum_{\ell=0}^{m} \alpha_{\ell}\|\omega\|^{2 \ell}, \quad \omega \in \mathbb{R}^{d} .
$$

It is trivial to observe that

$$
\widehat{L}(\omega)>\|\omega\|^{2 m}, \quad \omega \in \mathbb{R}^{d} .
$$

The operator $L$ defines a subspace $H^{m, L}$ of $L_{2}\left(\mathbb{R}^{d}\right)$. Namely $H^{m, L}$ is the space of distributions on $\mathbb{R}^{d}$ whose all derivatives up to order $m$ are in $L^{2}\left(\mathbb{R}^{d}\right)$, endowed with the following scalar product

$$
(f, g)_{m, L}=\int_{\mathbb{R}^{d}}\left(\sum_{\ell=0}^{m} \alpha_{\ell}\left(D^{\ell} f\right)(x) \cdot\left(D^{\ell} g\right)(x)\right) d x,
$$

and the associated norm

$$
\|f\|_{m, L}=\left(\int_{\mathbb{R}^{d}} \sum_{\ell=0}^{m} \alpha_{\ell}\left\|\left(D^{\ell} f\right)(x)\right\|^{2} d x\right)^{\frac{1}{2}} .
$$

Note that these are a scalar product and a norm since all $\alpha_{\ell}$ are positive numbers.

The norm $\|\cdot\|_{m, L}$ is equivalent to the norm $\|\cdot\|_{m}(2.1)$ of $H^{m}$. As a consequence of this, the elements of $H^{m, L}$ are actually continuous functions and $H^{m, L}$, endowed with the norm $\|\cdot\|_{m, L}$ is a Hilbert space. Furthermore, if $A$ is a finite set of elements in $\mathbb{R}^{d}$, and if $\left(\lambda_{a}\right)_{a \in A}$ are real numbers, then the application $f \in H^{m, L} \longrightarrow \sum_{a \in A} \lambda_{a} f(a)$ is a linear and continuous functional on $H^{m, L}$. 
3.2 Minimization property

Theorem 3.1 Let $A$ be a (finite or infinite) set of elements in $\mathbb{R}^{d}$.

(i) Suppose there exists a function $g$ in $H^{m, L}$ such that $\forall a \in A, g(a)=y_{a}$. Then the set of all functions $f$ in $H^{m, L}$ meeting $\forall a \in A, f(a)=y_{a}$ has a unique element with minimal norm $\|f\|_{m, L}$.

(ii) Suppose $A$ is a finite set. Then there exist various functions $g$ in $H^{m, L}$ such that $\forall a \in A, g(a)=y_{a}$, and so, from $i$ ), the sets of all functions $f$ in $H^{m, L}$ meeting $\forall a \in A, f(a)=y_{a}$ has a unique element with minimal norm $\|f\|_{m, L}$.

Proof Since for any $a$ in $A$, the functional $a \longrightarrow f(a)$ is continuous on $H^{m, L}$, the set of functions $f$ in $H^{m, L}$ such that $f(a)=y_{a}$ is a closed convex set. As a consequence the set of functions $f$ in $H^{m, L}$ such that $\forall a \in A, f(a)=y_{a}$ is a closed convex set (as an intersection of closed convex sets). Obviously this set is nonempty (for example, if $R<\min _{a \in A} \min _{b \in A \backslash\{a\}}\|b-a\|$, the function $f$ defined by $\forall x \in \mathbb{R}^{d}, f(x)=\sum_{a \in A} y_{a} \varphi(x-a)$ where the function $\varphi$ is defined by $\varphi(x)=\exp \left(-\frac{\|x\|^{2}}{\|x\|^{2}-R^{2}}\right)$ if $\|x\|<R$ and $\varphi(x)=0$ if $\|x\| \geq R$, is in this set).

As a consequence of it, there exists one and only one projection of 0 onto this closed convex set, which is precisely the unique element in $H^{m, L}$ of minimal norm $\|\cdot\|_{m, L}$ interpolating the points $\left(a, y_{a}\right)_{a \in A}$.

In the sequel, for a given finite set $A \subset \mathbb{R}^{d}$ and a given vector $y=\left(y_{a}\right)_{a \in A}$ $\left(y_{a} \in \mathbb{R}\right)$, the set of functions in $H^{m, L}$ meeting $\forall a \in A, f(a)=y_{a}$ is denoted by $H_{A, y}^{m, L}$, or $H_{A, 0}^{m, L}$ when all $y_{a}$ are zero, and the element of $H_{A, y}^{m, L}$ of minimal norm is denoted by $\sigma_{A, y}^{m, L}$, or more simply by $\sigma$ whenever there is no ambiguity. Furthermore $\operatorname{Dirac}(a)$ means the Dirac distribution in $a$.

Theorem 3.2 With the above notations, there exist real numbers $\left(\lambda_{a}\right)_{a \in A}$ such that $\sigma$ meets the relation $L(\sigma)=\sum_{a \in A} \lambda_{a} \delta_{a}$.

Proof Clearly $H_{A, 0}^{m, L}$ is a vectorial subspace of $H^{m, L}$. Furthermore if $f$ and $g$ are in $H_{A, y}^{m, L}, f-g$ is in $H_{A, 0}^{m, L}$. Now, let $f_{y}$ be an arbitrary function in $H_{A, y}^{m, L}$. Then any function $g$ in $H_{A, y}^{m, L}$ can be written in the form $g=f_{y}+v$ where $v$ is some function in $H_{A, 0}^{m, L}$. Since $\sigma$ is the orthogonal projection of 0 on $H_{A, y}^{m, L}$ is also orthogonal to the "translated" space $H_{A, 0}^{m, L}$, and so for any $y$, and any $v$ in $H_{A, 0}^{m, L}$ we have the relation $(\sigma, v)_{m, L}=0$.

Let us arbitrarily choose some functions $\left(\Lambda_{a}\right)_{a \in A}$ in $H^{m, L}$ meeting $\Lambda_{a}(a)=$ 1 and $\forall b \in A \backslash\{a\}, \Lambda_{a}(b)=0$, and let $\lambda_{a}=\left(\sigma, \Lambda_{a}\right)_{m, L}$.

For any function $\varphi$ in $H^{m, L}$, let now $g=\varphi-\sum_{a \in A} \varphi(a) \Lambda_{a} \cdot g$ is in $H_{A, 0}^{m, L}$, and so $(\sigma, g)_{m, L}=0$, which is $(\sigma, \varphi)_{m, L}=\sum_{a \in A} \varphi(a)\left(\sigma, \Lambda_{a}\right)_{m, L}$ and so $(\sigma, \varphi)_{m, L}=\sum_{a \in A} \lambda_{a} \varphi(a)$. Since any function in the set $\mathcal{H}$ of compactly 
supported, indefinitely differentiable functions is in $H^{m, L}$, this is true for any function $\varphi$ in $\mathcal{H}$, which is, $\langle\cdot, \cdot\rangle_{\mathcal{H}}$ being the distribution duality product,

$$
(\sigma, \varphi)_{m, L}=\sum_{a \in A} \lambda_{a} \varphi(a)=<\sum_{a \in A} \lambda_{a} \operatorname{Dirac}(a), \varphi>_{\mathcal{H}}
$$

Now we also have

$$
\begin{aligned}
(\sigma, \varphi)_{m, L} & =\int_{\mathbb{R}^{d}}\left(\sum_{i=1}^{m} \alpha_{i}\left(D^{i} \sigma\right)(x) \cdot\left(D^{i} \varphi\right)(x)\right) d x \\
& =\sum_{i=1}^{m} \alpha_{i}<D^{i} \sigma, D^{i} \varphi>_{\mathcal{H}} \\
& =<\sum_{i=1}^{m} \alpha_{i}(-\Delta)^{i} \sigma, \varphi>_{\mathcal{H}} \\
& =<L \sigma, \varphi>_{\mathcal{H}}
\end{aligned}
$$

So we have $L \sigma=\sum_{i=1}^{m} \lambda_{a} \operatorname{Dirac}(a)$, which ends the proof.

Now we can state

Theorem 3.3 For any finite set $A \subset \mathbb{R}^{d}$ and any vector $y=\left(y_{a}\right)_{a \in A}, \sigma$ being the unique function in $H^{m, L}$ minimizing $\|f\|_{m, L}$ among all functions $f$ such that $f(a)=y_{a}$, there exists a unique vector $\lambda=\left\{\lambda_{a}\right\}_{a \in A}$ such that $\sigma=\sum_{a \in A} \lambda_{a} u(\cdot-a)$.

Proof The existence of $\lambda$ is a direct consequence of Theorem 3.2 and of the relation $L u=$ Dirac defining the function $u$.

Note that this also means that the function $s=\sum_{b \in A} \lambda_{b} u(\cdot-b)$ is such that for any $a$ in $A, s(a)=y_{a}$, is the unique function which minimizes $L f$ under the condition $f(a)=y_{a}$ for any $a$ in $A$.

\section{Multiresolution Analysis and $L$ pre-wavelets}

The matrix $N$ was defined just after equation (2.2) as the transpose of the matrix $M$. We consider an acceptable dilation matrix $M$ for $\mathbb{Z}^{d}$ which is also a similarity ( i.e. $M=\rho M_{0}$, where $M_{0}$ is an orthogonal matrix and $\left|\rho^{d}\right|=$ $|\operatorname{det}(M)|$ is an integer $\geq 2$ ).

\subsection{Multiresolution analysis}

Let us denote by $V_{j}$ the $L^{2}\left(\mathbb{R}^{d}\right)$-closure of the set generated by all linear combinations of the $M^{-j} Z^{d}$ translates of $u$ :

$$
V_{j}=\overline{\left\{\sum_{l \in \mathbb{Z}^{d}} v_{l}^{j} u\left(\cdot-M^{-j} l\right), v \in l^{2}\left(\mathbb{Z}^{d}\right)\right\}}
$$


Proposition 4.1 The set $\left\{u\left(\cdot-M^{-j} l\right)\right\}_{l \in \mathbb{Z}^{d}}$ is a Riesz basis for $V_{j}$, i.e. there exist two constants $0<\mathcal{A}_{j} \leq \mathcal{B}_{j}<\infty$ such that for any $\omega \in \mathbb{R}^{d}$

$$
\mathcal{A}_{j} \leq \sum_{l \in \mathbb{Z}^{d}}\left|\widehat{u}\left(\omega+2 \pi N^{j} l\right)\right|^{2} \leq \mathcal{B}_{j}
$$

Proof Due to the decay and positivity of $\widehat{u}(\omega)$, the series (4.1) is convergent and strictly positive, so the constants $\mathcal{A}_{j}$ and $\mathcal{B}_{j}$ exist, and in the following we provide an estimate of them. Since the series (4.1) is $2 \pi N^{j}$-periodic, we only study the case $\omega \in \Omega_{j}$.

For the lower bound, by using the fact that $\widehat{u}(\omega)=\widehat{L}^{-1}(\omega)$ and (3.2), we get

$$
\sum_{l \in \mathbb{Z}^{d}}\left|\widehat{u}\left(\omega+2 \pi N^{j} l\right)\right|^{2}>\widehat{u}^{2}(\omega) \geq \frac{1}{C^{2}\left(1+\|\omega\|^{2}\right)^{2 m}} .
$$

Since $\omega \in \Omega_{j}$, and $N$ is a similarity, we have that $\|\omega\| \leq \pi\left\|N^{j} k\right\|$, i.e. $\|\omega\| \leq$ $|\operatorname{det}(M)|^{j / d} \pi\|k\|, \forall k \in \mathbb{Z}^{d} \backslash\{0\}$. Let us take $\|k\|=1$, then

$$
\sum_{l \in \mathbb{Z}^{d}}\left|\widehat{u}\left(\omega+2 \pi N^{j} l\right)\right|^{2}>\frac{1}{C^{2}\left(1+\pi^{2}|\operatorname{det}(M)|^{2 j / d}\right)^{2 m}}=: \mathcal{A}_{j} .
$$

In particular, for $j>0$

$$
\left(1+\pi^{2}|\operatorname{det}(M)|^{2 j / d}\right)^{2 m} \leq\left(2 \pi^{2}|\operatorname{det}(M)|^{2 j / d}\right)^{2 m}=2^{2 m} \pi^{4 m}|\operatorname{det}(M)|^{4 m j / d}
$$

and then

$$
\sum_{l \in \mathbb{Z}^{d}}\left|\widehat{u}\left(\omega+2 \pi N^{j} l\right)\right|^{2}>C_{0}|\operatorname{det}(M)|^{-4 m j / d}=: \mathcal{A}_{j}^{0},
$$

where $C_{0}=2^{-2 m} \pi^{-4 m} C^{-2}$.

Let now consider the upper bound. For any $\omega \in \Omega_{j}$, using (3.2) and (3.8) we can write

$$
\begin{aligned}
\sum_{l \in \mathbb{Z}^{d}}\left|\widehat{u}\left(\omega+2 \pi N^{j} l\right)\right|^{2} & =|\widehat{u}(\omega)|^{2}+\sum_{l \in \mathbb{Z}^{d}, l \neq 0}\left|\widehat{u}\left(\omega+2 \pi N^{j} l\right)\right|^{2} \\
& \leq \prod_{\ell=1}^{m} \kappa_{\ell}^{-4}+\sum_{l \in \mathbb{Z}^{d}, l \neq 0}\left(\left\|\omega+2 \pi N^{j} l\right\|\right)^{-4 m}
\end{aligned}
$$

Since $l \neq 0,\|\omega\| \leq|\operatorname{det}(M)|^{j / d} \pi$, and

$$
\left\|\omega+2 \pi N^{j} l\right\| \geq\left|\|\omega\|-\left\|2 \pi N^{j} l\right\|\right|=2 \pi|\operatorname{det}(M)|^{j / d}\|l\|-\|\omega\|,
$$

we get

$$
\left\|\omega+2 \pi N^{j} l\right\| \geq 2 \pi|\operatorname{det}(M)|^{j / d}\left(\|l\|-\frac{1}{2}\right) \geq \pi|\operatorname{det}(M)|^{j / d} n
$$


where $n=\|l\|_{\infty}$. Let $D_{n}$ be the set $\left\{l \in \mathbb{Z}^{d} \mid\|l\|_{\infty}=n\right\}$, its cardinality $\left|D_{n}\right|$ is such that $\left|D_{n}\right| \leq 2^{2 d-1} n^{d-1}$, then (4.3) becomes

$$
\begin{aligned}
\sum_{l \in \mathbb{Z}^{d}}\left|\widehat{u}\left(\omega+2 \pi N^{j} l\right)\right|^{2} & \leq \prod_{\ell=1}^{m} \kappa_{\ell}^{-4}+\pi^{-4 m}|\operatorname{det}(M)|^{-4 m j / d} \sum_{l \in \mathbb{Z}^{d}, l \neq 0}\left(\|l\|_{\infty}\right)^{-4 m} \\
& \leq \prod_{\ell=1}^{m} \kappa_{\ell}^{-4}+2^{2 d-1} \pi^{-4 m}|\operatorname{det}(M)|^{-4 m j / d} \sum_{n=1}^{\infty} n^{-4 m+d-1}
\end{aligned}
$$

Since $m>d / 2$, the series $\sum_{n=1}^{\infty} n^{-4 m+d-1}$ is convergent to $\zeta(4 m-d+1)$ where $\zeta$ is the Riemann zeta function $\zeta(r):=\sum_{n=1}^{\infty} n^{-r}$. Then

$$
\sum_{l \in \mathbb{Z}^{d}}\left|\widehat{u}\left(\omega+2 \pi N^{j} l\right)\right|^{2} \leq \prod_{\ell=1}^{m} \kappa_{\ell}^{-4}+2^{2 d-1} \pi^{-4 m} \zeta(4 m-d+1)|\operatorname{det}(M)|^{-4 m j / d}:=\mathcal{B}_{j} .
$$

Proposition 4.2 The spaces $\left\{V_{j}\right\}_{j \in \mathbb{Z}}$ form a non-stationary multiresolution analysis of $L^{2}\left(\mathbb{R}^{d}\right)$.

Proof Let us consider the definition (2.1). Property (4) is a direct consequence of the definition of $V_{j}$ and (3) has been proved in Proposition 4.1. Property (1) follows from the definition of the dilation matrix $M$ and the Riesz condition (4.1). Since Supp $\widehat{u}=\mathbb{R}^{d}$, property (2) (density in $L^{2}\left(\mathbb{R}^{d}\right)$ ) comes from $[8$, Theorem 4.3]. Finally property (3) comes again from a general results in [8].

The function $u$ does not satisfy any (non-stationary) refinable equation, then in order to set up a NSMRA, we consider the cardinal function $\Lambda_{j}$ of $V_{j}$, which, in our case, always exists, and generates a Riesz basis for $V_{j}$. We will show that it satisfies a non-stationary refinement equation which gives in the time domain and for each level $j$ the so called recontruction coefficients.

\subsubsection{The cardinal function}

As remarked in Section 3.1, the function $u$ decays exponentially, then $u \in$ $L^{1}\left(\mathbb{R}^{d}\right)$, and the sequence $\beta^{j}=\left\{\beta_{l}^{j}\right\}=\left\{u\left(M^{-j} l\right)\right\}_{l \in \mathbb{Z}^{d}}$ has an exponential decay and belongs to $\ell^{1}\left(\mathbb{Z}^{d}\right)$. As a consequence the trigonometric series

$$
\widehat{\beta^{j}}(\omega)=\sum_{l \in \mathbb{Z}^{d}} u\left(M^{-j} l\right) e^{-i \omega \cdot M^{-j} l}
$$

is absolutely convergent and by using the Poisson summation formula, we have

$$
\sum_{l \in \mathbb{Z}^{d}} u\left(M^{-j} l\right) e^{-i \omega \cdot M^{-j} l}=|\operatorname{det}(M)|^{j} \sum_{l \in \mathbb{Z}^{d}} \widehat{u}\left(\omega-2 \pi N^{j} l\right) .
$$


Since $\widehat{u}$ is strictly positive, also $\widehat{\beta^{j}}(\omega)$ is strictly positive. Then by Wiener's lemma, there are unique absolutely summable coefficients $a^{j}=\left\{a_{l}^{j}\right\}_{l \in \mathbb{Z}^{d}}$ such that the cardinal function

$$
\Lambda_{j}=\sum_{l \in \mathbb{Z}^{d}} a_{l}^{j} u\left(\cdot-M^{-j} l\right)
$$

satisfies $\Lambda_{j}\left(M^{-j} l\right)=\delta_{0 l}, l \in \mathbb{Z}^{d}$. The vector $a^{j}$ is such that
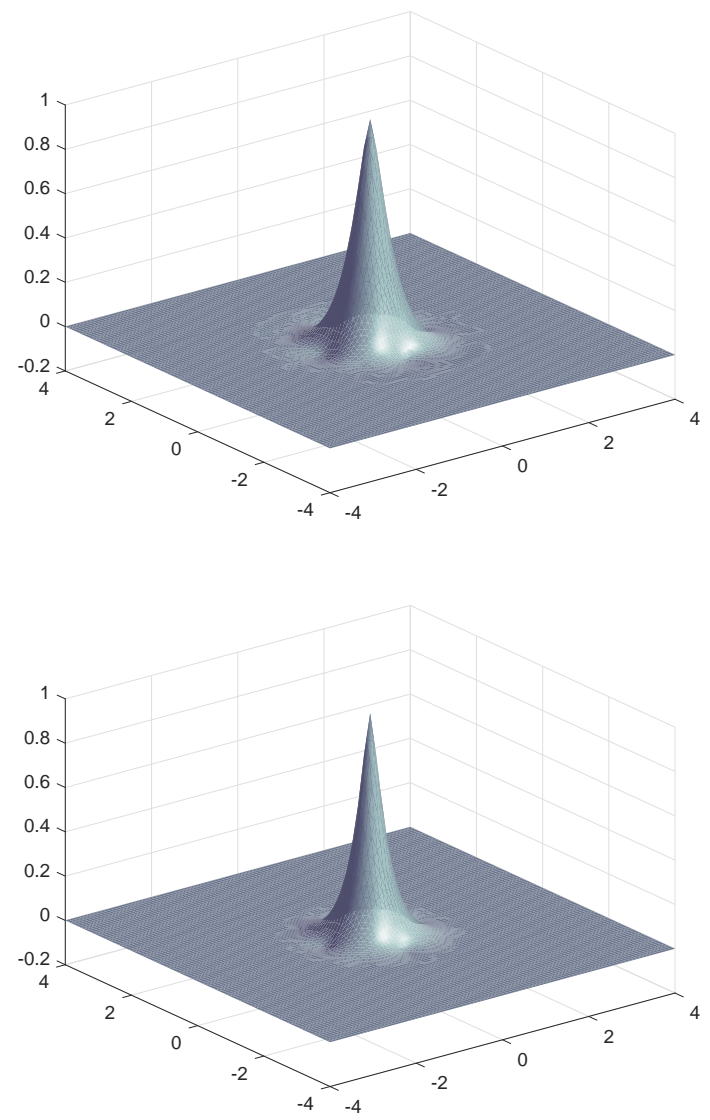

Fig. 4.1 Top: $\Lambda_{0}$ with $m=2, \kappa_{1}=3, \kappa_{2}=4$. Bottom: $\Lambda_{0}$ with $\kappa_{1}=3, \kappa_{2}=7$.

$$
a^{j} * \beta^{j}=\delta,
$$

that is

$$
\widehat{a^{j}}(\omega) \widehat{\beta^{j}}(\omega)=1
$$


and

$$
\widehat{a^{j}}(\omega)=\sum_{l \in \mathbb{Z}^{d}} a_{l}^{j} e^{-i \omega \cdot M^{-j} l}=\frac{1}{|\operatorname{det}(M)|^{j} \sum_{l \in \mathbb{Z}^{d}} \widehat{u}\left(\omega-2 \pi N^{j} l\right)} .
$$

Moreover (see e.g [2, Lemma 2]), $a^{j}$ has the same decay as $\beta^{j}$, that is it decays exponentially. The Fourier transform of $\Lambda_{j}$ takes the form

$$
\widehat{\Lambda_{j}}(\omega)=\frac{\widehat{u}(\omega)}{\widehat{\beta^{j}}(\omega)}=\frac{\widehat{u}(\omega)}{|\operatorname{det}(M)|^{j} \sum_{l \in \mathbb{Z}^{d}} \widehat{u}\left(\omega-2 \pi N^{j} l\right)} .
$$

Proposition 4.3 The cardinal function $\Lambda_{j}$ is such that:

(i) $\widehat{\Lambda}_{j}(\omega)=\frac{|\operatorname{det}(M)|^{-j}}{1+\sum_{l \in \mathbb{Z}^{d} \backslash\{0\}} \frac{\hat{L}(\omega)}{\hat{L}\left(\omega-2 \pi N^{j} l\right)}}$;

(ii) $\Lambda_{j}(x)$ decays exponentially as $x \rightarrow \infty$.

Proof In terms of $\widehat{L},(4.9)$ becomes

$$
\widehat{\Lambda_{j}}(\omega)=\frac{(\widehat{L}(\omega))^{-1}}{|\operatorname{det}(M)|^{j} \sum_{l \in \mathbb{Z}^{d}}\left(\widehat{L}\left(\omega-2 \pi N^{j} l\right)\right)^{-1}},
$$

or

$$
\widehat{\Lambda_{j}}(\omega)=\frac{|\operatorname{det}(M)|^{-j}}{1+\sum_{l \in \mathbb{Z}^{d} \backslash\{0\}} \frac{\widehat{L}(\omega)}{\widehat{L}\left(\omega-2 \pi N^{j} l\right)}} .
$$

The function $\widehat{\Lambda_{j}}$ is infinitely differentiable in zero, then $\Lambda_{j}$ is exponentially decaying at infinity.

Also the $M^{-j} \mathbb{Z}^{d}$ translates of $\Lambda_{j}$ are stable and generate a Riesz basis for $V_{j}$.

Proposition 4.4 There exists a constant $0<\mathcal{L}_{j}<|\operatorname{det}(M)|^{-2 j}$ such that

$$
\mathcal{L}_{j} \leq \sum_{l \in \mathbb{Z}^{d}}\left|\widehat{\Lambda_{j}}\left(\omega+2 \pi N^{j} l\right)\right|^{2} \leq|\operatorname{det}(M)|^{-2 j} .
$$

Proof By using (4.9), and using the fact that $\widehat{\beta^{j}}(\omega)$ is $2 \pi N^{j} \mathbb{Z}^{d}$-periodic, we get

$$
\begin{aligned}
\sum_{l \in \mathbb{Z}^{d}}\left|\widehat{\Lambda}_{j}\left(\omega+2 \pi N^{j} l\right)\right|^{2} & =\frac{\sum_{l \in \mathbb{Z}^{d}}\left|\widehat{u}\left(\omega+2 \pi N^{j} l\right)\right|^{2}}{|\operatorname{det}(M)|^{2 j}\left(\sum_{l \in \mathbb{Z}^{d}}\left|\widehat{u}\left(\omega+2 \pi N^{j} l\right)\right|\right)^{2}} \\
& <|\operatorname{det}(M)|^{-2 j} .
\end{aligned}
$$

For the lower bound, we proceed as in Proposition 4.1, and we can state that

$$
\widehat{\beta^{j}}(\omega) \leq \prod_{\ell=1}^{m} \kappa_{\ell}^{-2}+2^{2 d-1} \pi^{-2 m} \zeta(2 m-d+1)|\operatorname{det}(M)|^{-2 m j / d}:=\mathcal{B}_{\beta_{j}} .
$$


then

$$
\sum_{l \in \mathbb{Z}^{d}}\left|\widehat{\Lambda_{j}}\left(\omega+2 \pi N^{j} l\right)\right|^{2} \geq \frac{\mathcal{A}_{j}}{\mathcal{B}_{\beta_{j}}^{2}}:=\mathcal{L}_{j}>0
$$

The Lagrange function $\Lambda_{j}$ satisfies a non-stationary refinement equation.

Proposition 4.5 The $2 \pi N^{j+1}$-periodic function $\widehat{c^{j}}$ defined by

$$
\widehat{c^{j}}(\omega):=|\operatorname{det}(M)| \frac{\sum_{l \in \mathbb{Z}^{d}} \widehat{u}\left(\omega-2 \pi N^{j+1} l\right)}{\sum_{l \in \mathbb{Z}^{d}} \widehat{u}\left(\omega-2 \pi N^{j} l\right)}, \quad \omega \in \mathbb{R}^{d}
$$

meets

$$
\widehat{\Lambda_{j}}=\widehat{c^{j}} \widehat{\Lambda_{j+1}} \text {, }
$$

and belongs to $L^{2}\left(\Omega_{j}\right)$. . The corresponding vector $c^{j}=\left\{c_{l}^{j}\right\}_{l \in \mathbb{Z}^{d}}$ is such that

$$
\Lambda_{j}=\sum_{l \in \mathbb{Z}^{d}} c_{l}^{j} \Lambda_{j+1}\left(\cdot-M^{-j-1} l\right) .
$$

The reconstruction coefficients $\left(c_{k}^{j}\right)_{k \in \mathbb{Z}^{d}}$ decay exponentially and

$$
c_{l}^{j}=\Lambda_{j}\left(M^{-j-1} l\right), \quad l \in \mathbb{Z}^{d}, \quad j \in \mathbb{Z} .
$$

Proof Since from (4.9)

$$
\widehat{\Lambda_{j}}(\omega)=\frac{\widehat{u}(\omega)}{|\operatorname{det}(M)|^{j} \sum_{l \in \mathbb{Z}^{d}} \widehat{u}\left(\omega-2 \pi N^{j} l\right)},
$$

and

we get that

$$
\widehat{\Lambda_{j+1}}(\omega)=\frac{\widehat{u}(\omega)}{|\operatorname{det}(M)|^{j+1} \sum_{l \in \mathbb{Z}^{d}} \widehat{u}\left(\omega-2 \pi N^{j+1} l\right)},
$$

$$
\widehat{\Lambda_{j}}(\omega)=|\operatorname{det}(M)| \frac{\sum_{l \in \mathbb{Z}^{d}} \widehat{u}\left(\omega-2 \pi N^{j+1} l\right)}{\sum_{l \in \mathbb{Z}^{d}} \widehat{u}\left(\omega-2 \pi N^{j} l\right)} \widehat{\Lambda_{j+1}}(\omega)=\widehat{c^{j}}(\omega) \widehat{\Lambda_{j+1}}(\omega) .
$$

The $2 \pi N^{j}$ periodic function $\widehat{c^{j}}(\omega)$ is bounded and then is in $L^{2}\left(\Omega_{j}\right)$. It can be expressed in terms of its Fourier series, i.e.

$$
\widehat{c^{j}}(\omega)=\sum_{l \in \mathbb{Z}^{d}} c_{l}^{j} e^{-i \omega \cdot M^{-j-1} l}
$$

then (4.13) holds. Using the cardinal conditions, the vector $c^{j}=\left\{c_{l}^{j}\right\}_{l \in \mathbb{Z}^{d}}$ is such that

$$
c_{l}^{j}=\Lambda_{j}\left(M^{-j-1} l\right), \quad l \in \mathbb{Z}^{d}, \quad j \in \mathbb{Z},
$$

and as direct consequence of Proposition 4.3, $c^{j}$ decays exponentially.

We can use the Lagrangian functions $\Lambda_{j}$ in order to set up the NSMRA $\left\{V_{j}\right\}_{j \in \mathbb{Z}}$, associated with $\left(\mathbb{Z}^{d}, M\right)$. 
4.2 L-pre wavelets

As usual, we define the wavelet space $W_{j}$ as the orthogonal complement of $V_{j}$ in $V_{j+1}$

$$
V_{j+1}=V_{j} \bigoplus W_{j}
$$

It follows that the spaces $W_{j} \quad\left(V_{j+1}=V_{j} \bigoplus W_{j}\right)$ are mutually orthogonal, and then

$$
L^{2}\left(\mathbb{R}^{d}\right)=\bigoplus_{j \in \mathbb{Z}} W_{j}
$$

To define a set of pre-wavelets spanning $W_{j}$, we use a suitable differential operator as done for instance in [16]. Namely we consider

$$
L^{2}:=\prod_{\ell=1}^{m}\left(-\Delta+\kappa_{\ell}^{2} I\right)^{2},
$$

and its fundamental solution $u_{2}$, having Fourier transform $\widehat{u_{2}}$ meeting, for any $\omega$ in $\mathbb{R}^{d}$,

$$
\widehat{u_{2}}(\omega)=\prod_{\ell=1}^{m}\left(\|\omega\|^{2}+\kappa_{\ell}\right)^{-2}=\widehat{u}^{2}(\omega) .
$$

$\widehat{u_{2}}$ is infinitely differentiable in zero and then $u_{2}$ is exponentially decaying at infinity. The vector $\beta_{u_{2}}^{j}=\left\{u_{2}\left(M^{-j} l\right)\right\}_{l \in \mathbb{Z}^{d}}$ decays exponentially too, and then the cardinal function $\Lambda_{u_{2}, j}$ associated to $u_{2}$ exists since $u_{2} \in L^{1}\left(\mathbb{R}^{d}\right)$, $\beta_{u_{2}}^{j} \in \ell^{1}\left(\mathbb{Z}^{d}\right)$ and

$$
\widehat{\beta_{u_{2}}^{j}}(\omega)=\sum_{l \in \mathbb{Z}^{d}} u_{2}\left(M^{-j} l\right) e^{-i \omega \cdot M^{-j} l} \neq 0, \omega \in \mathbb{R}^{d} .
$$

Then (see Section 4.1.1) there are unique absolutely summable coefficients $a_{u_{2}}^{j}:=\left\{a_{u_{2}, l}^{j}\right\}_{l \in \mathbb{Z}^{d}}$ such that the function

$$
\Lambda_{u_{2}, j}=\sum_{l \in \mathbb{Z}^{d}} a_{u_{2}, l}^{j} u_{2}\left(\cdot-M^{-j} l\right) \operatorname{meets} \Lambda_{u_{2}, j}\left(M^{-j} l\right)=\delta_{0 l} .
$$

With the same argument used for $\Lambda_{j}$, those coefficients are such that

$$
a_{u_{2}}^{j} * \beta_{u_{2}}^{j}=\delta,
$$

$a_{u_{2}}^{j}$ decays exponentially, and the Fourier transform of $\Lambda_{u_{2}, j}$ meets

$$
\widehat{\Lambda_{u_{2}, j}}(\omega)=\frac{\widehat{u}^{2}(\omega)}{\widehat{\beta_{u_{2}}^{j}}(\omega)}=\frac{\widehat{u}^{2}(\omega)}{|\operatorname{det}(M)|^{j} \sum_{l \in \mathbb{Z}^{d}} \widehat{u}^{2}\left(\omega-2 \pi N^{j} l\right)}, \quad \omega \in \mathbb{R}^{d}
$$

Let us consider the function $\psi_{j}$ defined by

$$
\psi_{j}=L \Lambda_{u_{2}, j+1} .
$$


Its Fourier transform meets

$$
\begin{aligned}
\widehat{\psi_{j}}(\omega) & =\widehat{L}(\omega) \widehat{\Lambda_{u_{2}, j+1}}=\frac{\widehat{u}(\omega)}{\widehat{\beta_{u_{2}}^{j+1}}(\omega)} \\
& =\frac{\widehat{u}(\omega)}{|\operatorname{det}(M)|^{j+1} \sum_{s \in \mathbb{Z}^{d}} \widehat{u}^{2}\left(\omega-2 \pi N^{j+1} s\right)}, \quad \omega \in \mathbb{R}^{d} .
\end{aligned}
$$

Clearly $\psi_{j}$ is in $V_{j+1}$, actually

$$
\begin{aligned}
\psi_{j} & =L \Lambda_{u_{2}, j+1}=L \sum_{l \in Z^{d}} a_{u_{2}, l}^{j+1} u_{2}\left(\cdot-M^{-j-1} l\right) \\
& =\sum_{l \in \mathbb{Z}^{d}} a_{u_{2}, l}^{j+1} L u_{2}\left(\cdot-M^{-j-1} l\right) \\
& =\sum_{l \in \mathbb{Z}^{d}} a_{u_{2}, l}^{j+1} u\left(\cdot-M^{-j-1} l\right) .
\end{aligned}
$$

Since $u(\cdot)=\sum_{l \in \mathbb{Z}^{d}} \beta_{l}^{j+1} \Lambda_{j+1}\left(\cdot-M^{-j-1} l\right)$, we also get

$$
\psi_{j}=\sum_{l \in \mathbb{Z}^{d}} d_{l}^{j} \Lambda_{j+1}\left(\cdot-M^{-j-1} l\right),
$$

where $d^{j}=a_{u_{2}}^{j+1} * \beta^{j+1}$, has an exponential decay and meets $d_{k}^{j}=\psi^{j}\left(M^{-j-1} k\right)$, $\forall k \in \mathbb{Z}^{d}$.

Moreover the set $\left\{\psi^{j}\left(\cdot-M^{-j-1} k\right)\right\}_{k \in \mathbb{Z}^{d}}$ generates $V_{j+1}$, indeed from (4.22) we get $\widehat{\psi^{j}}=\widehat{a_{u_{2}}^{j+1}} \widehat{u}$, and so, using (4.18),

$$
\widehat{u}=\widehat{\beta_{u_{2}}^{j+1}} \widehat{\psi_{j}}
$$

and then any function in $V_{j+1}$ can written in terms of $M^{-j-1} \mathbb{Z}^{d}$ shifts of $\psi_{j}$.

For any $e \in \mathcal{E}_{0}\left(\mathcal{E}, \mathcal{E}_{0}\right.$ defined in Section 2.1), let us consider the functions $\psi_{j}^{e} \in V_{j+1}$ given by

$$
\psi_{j}^{e}=\psi_{j}\left(\cdot-M^{-j-1} e\right) .
$$

In the following propositions we show that the functions $\left\{\psi_{j}^{e}\right\}_{e \in \mathcal{E}_{0}}$ are prewavelets, i.e. they are orthogonal to $V_{j}$, provide a basis for $W_{j}$, and form a Riesz basis.

We remark that the vector $d^{j}$ and the function $\Lambda_{j+1}$ decay exponentially, then according to [2, Lemma 3] the pre-wavelet generator $\psi_{j}$ too has an exponential decay. Moreover since $u \in C^{2 m-d-1}\left(\mathbb{R}^{d}\right)$ and $d^{j} \in l^{1}\left(\mathbb{Z}^{d}\right)$, $\psi_{j} \in C^{2 m-d-1}\left(\mathbb{R}^{d}\right)$. Figure 4.2 shows the pre-wavelet generator $\psi_{j}$ for $d=2$, $m=2$, and different values of the parameters $\kappa_{1}, \kappa_{2}$. When the parameters increase, $\psi_{j}$ becomes more concentrated around the origin.

Proposition 4.6 For any $e \in \mathcal{E}_{0}$, and any $k \in \mathbb{Z}^{d}, \psi_{j}^{e}(\cdot-M k) \perp V_{j}$. 

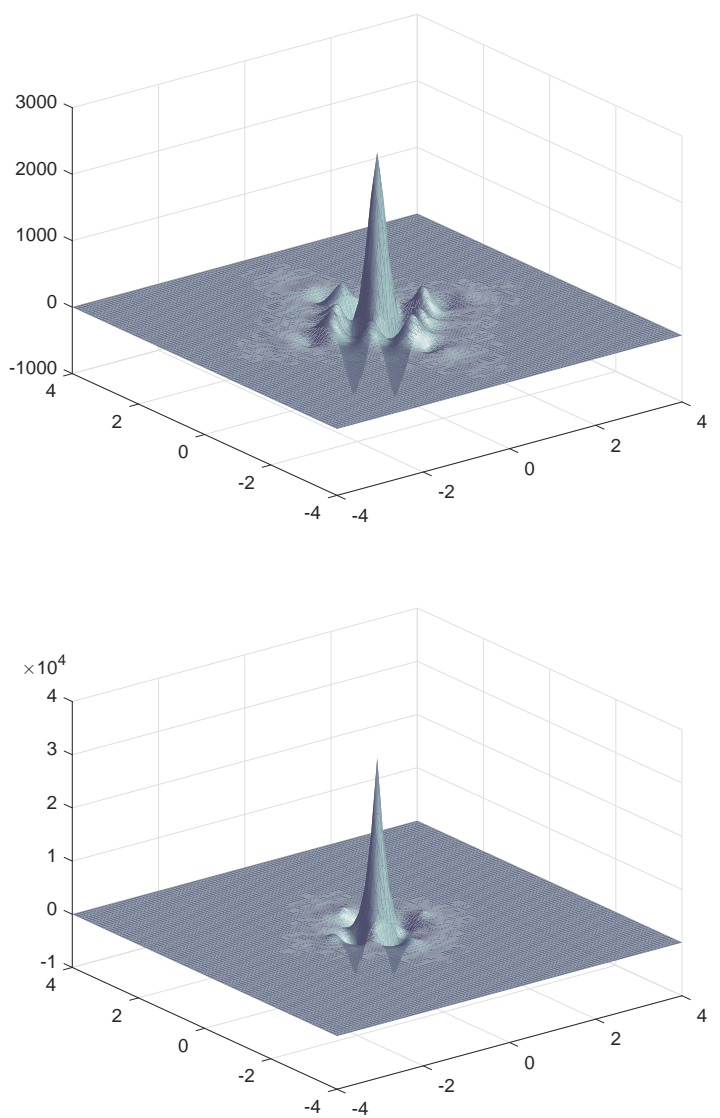

Fig. 4.2 Top: $\psi_{0}$ when $\kappa_{1}=3, \kappa_{2}=4$. Bottom: $\psi_{0}$ when $\kappa_{1}=3, \kappa_{2}=21$.

Proof Let $\sigma(x)=\sum_{k \in \mathbb{Z}^{d}} v_{k}^{j} u\left(\cdot-M^{-j} k\right)$ be a function of $V_{j}$ and let us consider the inner product $\left(\sigma, \psi_{j}^{e}\right)$. Using (3.6), the differential operator $L$ can be rewritten as

$$
L=\sum_{\ell=1}^{m} \alpha_{\ell}(-\Delta)^{\ell}+\alpha_{0} I:=L_{o}+\alpha_{0} I
$$

and $L u=$ Dirac is equivalent to

$$
L_{o} u=\operatorname{Dirac}-\alpha_{0} u .
$$


Then

$$
\begin{aligned}
\left(\sigma, \psi_{j}^{e}\right) & =\sum_{k \in \mathbb{Z}^{d}} v_{k}^{j}\left(u\left(\cdot-M^{-j} k\right), \psi_{j}\left(\cdot-M^{-j-1} e\right)\right) \\
& =\sum_{k \in \mathbb{Z}^{d}} v_{k}^{j}\left(u\left(\cdot-M^{-j} k\right), L \Lambda_{u_{2}, j+1}\left(\cdot-M^{-j-1} e\right)\right) \\
& =\sum_{k \in \mathbb{Z}^{d}} v_{k}^{j}\left(u\left(\cdot-M^{-j} k\right), L_{o} \Lambda_{u_{2}, j+1}\left(\cdot-M^{-j-1} e\right)\right) \\
& +\sum_{k \in \mathbb{Z}^{d}} v_{k}^{j}\left(u\left(\cdot-M^{-j} k\right), \alpha_{0} \Lambda_{u_{2}, j+1}\left(\cdot-M^{-j-1} e\right)\right) \\
& =\sum_{k \in \mathbb{Z}^{d}} v_{k}^{j}\left(L_{o} u\left(\cdot-M^{-j} k\right), \Lambda_{u_{2}, j+1}\left(\cdot-M^{-j-1} e\right)\right) \\
& +\sum_{k \in \mathbb{Z}^{d}} v_{k}^{j} \alpha_{0}\left(u\left(\cdot-M^{-j} k\right), \Lambda_{u_{2}, j+1}\left(\cdot-M^{-j-1} e\right)\right) .
\end{aligned}
$$

Let us denote by $\langle\cdot, \cdot\rangle$ the distribution duality product. By using (4.26) and, at the end, the fact that $M k-e$ is a non-zero multi-integer element while $\Lambda_{u_{2}, j+1}\left(M^{-j-1} l\right)$ is zero for any $l \in \mathbb{Z}^{d} \backslash\{0\}$, we get:

$$
\begin{aligned}
\left(\sigma, \psi_{j}^{e}\right) & =\sum_{k \in \mathbb{Z}^{d}} v_{k}^{j}<\operatorname{Dirac}\left(\cdot-M^{-j} k\right)-\alpha_{0} u\left(\cdot-M^{-j} k\right), \Lambda_{u_{2}, j+1}\left(\cdot-M^{-j-1} e\right)> \\
& +\sum_{k \in \mathbb{Z}^{d}} v_{k}^{j} \alpha_{0}<u\left(\cdot-M^{-j} k\right), \Lambda_{u_{2}, j+1}\left(\cdot-M^{-j-1} e\right)> \\
& =\sum_{k \in \mathbb{Z}^{d}} v_{k}^{j}<\operatorname{Dirac}\left(\cdot-M^{-j} k\right), \Lambda_{u_{2}, j+1}\left(\cdot-M^{-j-1} e\right)> \\
& =\sum_{k \in \mathbb{Z}^{d}} v_{k}^{j} \Lambda_{u_{2}, j+1}\left(M^{-j-1}(M k-e)\right) \\
& =0 .
\end{aligned}
$$

Proposition 4.7 The functions $\left\{\psi_{j}^{e}\right\}_{e \in \mathcal{E}_{0}}$ provide a basis for $W_{j}$.

Proof The support of $\widehat{\psi_{j}}$ is $\mathbb{R}^{d}, \psi_{j}$ generates $V_{j+1}$ and from Proposition 4.6 the functions $\left\{\psi_{j}^{e}\right\}_{e \in \mathcal{E}_{0}}$ are in $W_{j}$. Then according to [8, Theorem 3.14], the set $\left\{\psi_{j}^{e}\right\}_{e \in \mathcal{E}_{0}}$ is a basis for $W_{j}$.

Proposition 4.8 For any $j \in \mathbb{Z}$, the functions $\left\{\psi_{j}^{e}\right\}_{e \in \mathcal{E}_{0}}$ generate a Riesz basis for $W_{j}$.

Proof The family $\left\{\psi_{j}^{e}\left(\cdot-M^{-j} k\right)\right\}_{k \in \mathbb{Z}^{d}, e \in \mathcal{E}_{0}}$ form a Riesz basis of $W_{j}$ if for any function $f \in W_{j}$

$$
f(x)=\sum_{e \in \mathcal{E}_{0}} \sum_{k \in \mathbb{Z}^{d}} \mu_{k}^{j, e} \psi_{j}^{e}\left(x-M^{-j} k\right)
$$


where $\mu^{j, e}=\left\{\mu_{k}^{j, e}\right\}_{k \in \mathbb{Z}^{d}}, e \in \mathcal{E}_{0}$ belong to $\ell^{2}\left(\mathbb{Z}^{d}\right)$, there exist two positive real constants $0<\mathcal{C}_{j} \leq \mathcal{D}_{j}<\infty$ such that the following relation holds:

$$
\mathcal{C}_{j} \sum_{e \in \mathcal{E}_{0}}\left\|\mu^{j, e}\right\|^{2} \leq\left\|\sum_{e \in \mathcal{E}_{0}} \sum_{k \in \mathbb{Z}^{d}} \mu_{k}^{j, e} \psi_{j}^{e}\left(\cdot-M^{-j} k\right)\right\|^{2} \leq \mathcal{D}_{j} \sum_{e \in \mathcal{E}_{0}}\left\|\mu^{j, e}\right\|^{2} .
$$

Since the functions $\left\{\psi_{j}^{e}\right\}_{e \in \mathcal{E}_{0}}$ are linear independent, $f$ is the null function if and only if $\mu_{k}^{j, e}=0$ for any $k$ and $e$. In this case (4.27) trivially holds. Let us suppose that $f$ is not the null function. In this case its $L^{2}$-norm is greater than zero. Now (up to a constant) $\|f\|^{2}=\|\widehat{f}\|^{2}$, and

$$
\widehat{f}(\omega)=\sum_{e \in \mathcal{E}_{0}} \sum_{k \in \mathbb{Z}^{d}} \mu_{k}^{j, e} e^{-i \omega \cdot M^{-j} k} \widehat{\psi_{j}^{e}}(\omega) .
$$

Since $\psi_{j}^{e}=\psi_{j}\left(\cdot-M^{-j-1} e\right)$, we have $\widehat{\psi_{j}^{e}}=\widehat{\psi_{j}} e^{-i \omega \cdot M^{-j-1} e}$, and

$$
\begin{aligned}
\widehat{f}(\omega) & =\widehat{\psi_{j}}(\omega) \sum_{e \in \mathcal{E}_{0}} \sum_{k \in \mathbb{Z}^{d}} \mu_{k}^{j, e} e^{-i \omega \cdot M^{-j} k} e^{-i \omega \cdot M^{-j-1} e} \\
& =\widehat{\psi_{j}}(\omega) \sum_{e \in \mathcal{E}_{0}} \sum_{k \in \mathbb{Z}^{d}} \mu_{k}^{j, e} e^{-i \omega \cdot M^{-j-1}(M k+e)} .
\end{aligned}
$$

Let $\varsigma^{j}$ be the vector with components $\varsigma_{M k}^{j}=0, \varsigma_{M k-e}^{j}=\mu_{k}^{j, e}, e \in \mathcal{E}_{0}$, and $\widehat{\varsigma^{j}}(\omega)$ the convergent (in $L^{2}\left(\mathbb{R}^{d}\right)$ ) trigonometric series

$$
\widehat{\varsigma^{j}}(\omega)=\sum_{k \in \mathbb{Z}^{d}} \varsigma_{k}^{j} e^{-i \omega \cdot M^{-j-1} k} .
$$

Then

$$
\begin{aligned}
\widehat{f}(\omega) & =\widehat{\psi_{j}}(\omega) \sum_{e \in \mathcal{E}_{0}} \sum_{k \in \mathbb{Z}^{d}} \mu_{k}^{j, e} e^{-i \omega \cdot M^{-j-1}(M k-e)} \\
& =\widehat{\psi_{j}}(\omega) \sum_{k \in \mathbb{Z}^{d}} \varsigma_{k}^{j} e^{-i \omega \cdot M^{-j-1} k} \\
& =\widehat{\psi_{j}}(\omega) \widehat{\varsigma^{j}}(\omega),
\end{aligned}
$$

and

$$
\|f\|^{2}=\left\|\widehat{\psi_{j}} \widehat{\varsigma^{j}}\right\|^{2} .
$$

Using (2.5) and the $2 \pi N^{j+1} \mathbb{Z}^{d}$ periodicity of $\widehat{\varsigma^{j}}$, we have

$$
\begin{aligned}
\|f\|^{2} & =\int_{\mathbb{R}^{d}}\left|\widehat{\psi_{j}}(\omega)\right|^{2}\left|\widehat{\varsigma^{j}}(\omega)\right|^{2} d \omega \\
& =\sum_{\ell \in \mathbb{Z}^{d}} \int_{\Omega_{j+1}+2 \pi N^{j+1} \ell}\left|\widehat{\psi_{j}}(\omega)\right|^{2}\left|\widehat{\varsigma^{j}}(\omega)\right|^{2} d \omega \\
& =\int_{\Omega_{j+1}} \sum_{\ell \in \mathbb{Z}^{d}}\left|\widehat{\psi_{j}}\left(\omega+2 \pi N^{j+1} \ell\right)\right|^{2}\left|\widehat{\varsigma^{j}}(\omega)\right|^{2} d \omega .
\end{aligned}
$$


Remembering the expression $(4.22)$ of $\widehat{\psi_{j}}(\omega)$, the periodic function in $(4.34)$ is

$$
\sum_{\ell \in \mathbb{Z}^{d}}\left|\widehat{\psi_{j}}\left(\omega+2 \pi N^{j+1} \ell\right)\right|^{2}=\frac{\sum_{\ell \in \mathbb{Z}^{d}} \widehat{u}^{2}\left(\omega+2 \pi N^{j+1} \ell\right)}{|\operatorname{det}(M)|^{2 j+2}\left|\sum_{\ell \in \mathbb{Z}^{d}} \widehat{u_{2}}\left(\omega+2 \pi N^{j+1} \ell\right)\right|^{2}}
$$

By definition $\widehat{u_{2}}=\widehat{u}^{2}$, then

$$
\sum_{\ell \in \mathbb{Z}^{d}}\left|\widehat{\psi_{j}}\left(\omega+2 \pi N^{j+1} \ell\right)\right|^{2}=\frac{1}{|\operatorname{det}(M)|^{2 j+2} \sum_{\ell \in \mathbb{Z}^{d}} \widehat{u}^{2}\left(\omega+2 \pi N^{j+1} \ell\right)} .
$$

From (4.1), we obtain

$$
\frac{1}{|\operatorname{det}(M)|^{2 j+2} \mathcal{B}_{j+1}} \leq \sum_{\ell \in \mathbb{Z}^{d}}\left|\widehat{\psi_{j}}\left(\omega+2 \pi N^{j+1} \ell\right)\right|^{2} \leq \frac{1}{|\operatorname{det}(M)|^{2 j+2} \mathcal{A}_{j+1}}
$$

By applying the Parseval identity, we get

$$
\int_{\Omega_{j+1}}\left|\sum_{k \in \mathbb{Z}^{d}} \varsigma_{k}^{j} e^{i N^{-j-1} \omega \cdot k}\right|^{2} d \omega=\left|\Omega_{j+1}\right| \sum_{k \in \mathbb{Z}^{d}}\left|\varsigma_{k}^{j}\right|^{2}=|\operatorname{det}(M)|^{j+1} \sum_{k \in \mathbb{Z}^{d}}\left|\varsigma_{k}^{j}\right|^{2} .
$$

Then, using (4.36) we can bound (4.34) as follows

$$
\frac{1}{|\operatorname{det}(M)|^{j+1} \mathcal{B}_{j+1}} \sum_{k \in \mathbb{Z}^{d}}\left|\varsigma_{k}^{j}\right|^{2} \leq\|f\|^{2} \leq \frac{1}{|\operatorname{det}(M)|^{j+1} \mathcal{A}_{j+1}} \sum_{k \in \mathbb{Z}^{d}}\left|\varsigma_{k}^{j}\right|^{2}
$$

Remembering the definition of $\varsigma^{j}$, we obtain

$$
\mathcal{C}_{j} \sum_{e \in \mathcal{E}_{0}}\left\|\mu^{j, e}\right\|^{2} \leq\|f\|^{2} \leq \mathcal{D}_{j} \sum_{e \in \mathcal{E}_{0}}\left\|\mu^{j, e}\right\|^{2}
$$

where $\mathcal{C}_{j}=\frac{1}{|\operatorname{det}(M)|^{j+1} \mathcal{B}_{j+1}}$ and $\mathcal{D}_{j}=\frac{1}{|\operatorname{det}(M)|^{j+1} \mathcal{A}_{j+1}}$.

To ensure that the $L$-pre wavelets generate a Riesz basis for $L^{2}\left(\mathbb{R}^{d}\right)$, we need Riesz bounds independent on $j$. To guarantee this, we include in the wavelet decomposition a suitable multiresolution space $V_{J}$ with $J \geq 0$, i.e.

$$
L^{2}\left(\mathbb{R}^{d}\right)=V_{J} \bigoplus_{j>J} W_{j}
$$

and properly normalize the pre-wavelets $\psi_{j}^{e}$.

Theorem 4.1 For a given $J \geq 0$,

$$
\left\{\Lambda_{J}\left(\cdot-M^{-J} k\right)\right\}_{k \in \mathbb{Z}^{d}} \bigcup_{j>J, e \in \mathcal{E}_{0}}\left\{|\operatorname{det}(M)|^{-j(4 m / d-1)} \psi_{j}^{e}\left(\cdot-M^{-j} k\right)\right\}_{k \in \mathbb{Z}^{d}}
$$

form a Riesz basis for $L^{2}\left(\mathbb{R}^{d}\right)$. 
Proof We need to prove that for $j>J \geq 0$, the constants $\mathcal{C}_{j}$ and $\mathcal{D}_{j}$ are proportional to $|\operatorname{det}(M)|^{j(4 m / d-1)}$. Let us consider the upper bound. From (4.2), we obtain that for any $j>0, \mathcal{D}_{j}=\mathcal{D}_{0}|\operatorname{det}(M)|^{(4 m / d-1) j}$, where $\mathcal{D}_{0}=$ $C_{0}^{-1}|\operatorname{det}(M)|^{4 m / d-1}$. For the lower bound, we observe that

$$
\sum_{\ell \in \mathbb{Z}^{d}}\left|\widehat{\psi_{j}}\left(\omega+2 \pi N^{j+1} \ell\right)\right|^{2}=\sum_{e \in \mathcal{E}} \sum_{s \in \mathbb{Z}^{d}}\left|\widehat{\psi_{j}}\left(\omega+2 \pi N^{j+1}(e+N s)\right)\right|^{2},
$$

and that $\forall \bar{e} \in \mathcal{E}$

$$
\sum_{e \in \mathcal{E}} \sum_{s \in \mathbb{Z}^{d}}\left|\widehat{\psi_{j}}\left(\omega+2 \pi N^{j+1}(e+N s)\right)\right|^{2}>\sum_{s \in \mathbb{Z}^{d}}\left|\widehat{\psi_{j}}\left(\omega+2 \pi N^{j+1}(\bar{e}+N s)\right)\right|^{2} .
$$

We choose $\bar{e} \in \mathcal{E}_{0}$ and consider

$$
\sum_{s \in \mathbb{Z}^{d}}\left|\widehat{u}\left(\omega+2 \pi N^{j+1}(\bar{e}+N s)\right)\right|^{2} .
$$

We know that $\omega \in \Omega_{j+1}$ and that $N s+\bar{e} \neq 0$, then the points $\omega+2 \pi N^{j+1}(N s+$ $\bar{e}$ ) do not belong to $\Omega_{j+1}$. Considering that $\widehat{L}(t)>\|t\|^{2 m}$, (see (3.8)) we have $\widehat{u}(t)<\|t\|^{-2 m}$ for any $t \neq 0$. With the same arguments of the second part of Proposition 4.1 proof, we get

$$
\begin{aligned}
\sum_{s \in \mathbb{Z}^{d}}\left|\widehat{u}\left(\omega+2 \pi N^{j+1}(\bar{e}+N s)\right)\right|^{2} & <\sum_{s \in \mathbb{Z}^{d}}\left\|\omega+2 \pi N^{j+1}(\bar{e}+N s)\right\|^{-4 m} \\
& <C_{1}|\operatorname{det}(M)|^{-4 m(j+1) / d}
\end{aligned}
$$

where $C_{1}=2^{2 d-1} \pi^{-4 m} \zeta(4 m-d+1)$. Then

$$
\sum_{s \in \mathbb{Z}^{d}}\left|\widehat{\psi_{j}}\left(\omega+2 \pi N^{j+1} s\right)\right|^{2}>C_{1}^{-1}|\operatorname{det}(M)|^{4 m(j+1) / d}|\operatorname{det}(M)|^{-2 j-2} .
$$

So $\mathcal{C}_{j}=\mathcal{C}_{0}|\operatorname{det}(M)|^{(4 m / d-1) j}$, where $\mathcal{C}_{0}=C_{1}^{-1}|\operatorname{det}(M)|^{4 m / d-1}$.

The Riesz bounds for $\left\{|\operatorname{det}(M)|^{-j(4 m / d-1)} \psi_{j}^{e}\left(\cdot-M^{-j} k\right)\right\}_{k \in \mathbb{Z}^{d}}$ that generates $W_{j}, j>0$ do not depend on $j$ and then we get the proof.

\section{The filters}

In this section we give formulas for deriving the filters for performing the nonstationary wavelet decomposition and reconstruction. We do this in the time domain. Moreover we show that the filters belong to $\ell^{1}\left(\mathbb{Z}^{d}\right)$ and indeed they decay exponentially. 
5.1 The reconstruction filters

Both $\Lambda_{j} \in V_{j}$ and the pre-wavelets $\psi_{j}^{e} \in W_{j}$ are in $V_{j+1}$. The relations between $\Lambda_{j}, \psi_{j}$ and $\Lambda_{j+1}$ are given by (4.13) and (4.23). This means that there exist two vectors

$$
c^{j}=\left\{\Lambda_{j}\left(M^{-j-1} k\right)\right\}_{k \in \mathbb{Z}^{d}}, \quad d^{j}=a_{u_{2}}^{j+1} * \beta^{j+1},
$$

named reconstruction filters, such that the two non-stationary scale equations

$$
\begin{aligned}
& \Lambda_{j}=\sum_{k \in \mathbb{Z}^{d}} c_{k}^{j} \Lambda_{j+1}\left(\cdot-M^{-j-1} k\right):=c^{j} *_{j+1} \Lambda_{j+1} \\
& \psi_{j}=\sum_{k \in \mathbb{Z}^{d}} d_{k}^{j} \Lambda_{j+1}\left(\cdot-M^{-j-1} k\right):=d^{j} *_{j+1} \Lambda_{j+1}
\end{aligned}
$$

are satisfied. From (4.24), it follows

$$
\psi_{j}^{e}=\sum_{k \in \mathbb{Z}^{d}} d_{k}^{j} \Lambda_{j+1}\left(\cdot-M^{-j} k-M^{-j-1} e\right):=d^{j} *_{j+1} \Lambda_{j+1}\left(\cdot-M^{-j-1} e\right), \quad e \in \mathcal{E}_{0} .
$$

5.2 The decomposition filters

Since all the functions $\left\{\Lambda_{j}\left(\cdot-M^{-j-1} e\right)\right\}_{e \in \mathcal{E}}$ are in $V_{j+1}=V_{j} \oplus W_{j}$, and the family of functions $\left\{\Lambda_{j}\left(\cdot-M^{-j} k\right)\right\}_{k \in \mathbb{Z}^{d}}$ and $\left\{\psi_{j}^{e}\left(\cdot-M^{-j} k\right)\right\}_{k \in \mathbb{Z}^{d}, e \in \mathcal{E}_{0}}$ are basis of $V_{j}$ and $W_{j}$ respectively, for any $e \in \mathcal{E}$ there exist vectors in $l^{2}\left(\mathbb{Z}^{d}\right)$, which we denote by $p^{j, e}$ and $q^{j, e, e^{\prime}}\left(e^{\prime} \in \mathcal{E}_{0}\right)$ such that

$$
\Lambda_{j+1}\left(\cdot-M^{-j-1} e\right)=p^{j, e} *_{j} \Lambda_{j}+\sum_{e^{\prime} \in \mathcal{E}_{0}} q^{j, e, e^{\prime}} *_{j} \psi_{j}^{e}, \quad e \in \mathcal{E}
$$

The decomposition filters $p^{j}$ and $q^{j, e^{\prime}}\left(e^{\prime} \in \mathcal{E}_{0}\right)$ are defined for all $k \in \mathbb{Z}^{d}$ and $e \in \mathcal{E}$ by

$$
p_{M k-e}^{j}=p_{k}^{j, e}, \quad \text { and } \quad q_{M k-e}^{j, e^{\prime}}=q_{k}^{j, e, e^{\prime}}, e^{\prime} \in \mathcal{E}_{0} .
$$

In order to compute the decomposition filters, we follow [2]. Let us consider first the decomposition filter $p^{j}$ as defined by (5.5). The following proposition gives the formulae for deriving $p^{j, e}$ from the vectors $c^{j}, \beta_{u_{2}}^{j+1}$ and $d^{j}$. In order to do this we observe that the convolution $\Lambda_{j} * \Lambda_{j}$ can be expressed in terms of $\Lambda_{j, u_{2}}$.

Lemma 5.1 There exist an exponentially decaying vector $\gamma^{j}$ such that

$$
\Lambda_{j} * \Lambda_{j}=\sum_{\ell \in \mathbb{Z}^{d}} \gamma_{\ell}^{j} \Lambda_{u_{2}, j}\left(\cdot-M^{-j} \ell\right):=\gamma^{j} *_{j} \Lambda_{u_{2}, j}
$$


It takes the expression

$$
\gamma^{j}=a^{j} * a^{j} * \beta_{u_{2}}^{j}
$$

and the vector

$$
\gamma^{-j}:=a_{u_{2}}^{j} * \beta^{j} * \beta^{j} \text { is such that } \gamma^{j} * \gamma^{-j}=\delta .
$$

Moreover

$$
\gamma_{\ell}^{j}=\left(\Lambda_{j} * \Lambda_{j}\right)\left(M^{-j} \ell\right) .
$$

Proof Using (4.5) and (4.19)

$$
{\widehat{\Lambda_{j} * \Lambda_{j}}}(\omega)=\left(\widehat{\Lambda_{j}}(\omega)\right)^{2}=\left(\widehat{a^{j}}(\omega)\right)^{2}(\widehat{u}(\omega))^{2},
$$

and

$$
\widehat{u}_{2}(\omega)=(\widehat{u}(\omega))^{2}=\widehat{\beta_{u_{2}}^{j}} \widehat{\Lambda_{u_{2}, j}}(\omega) .
$$

Then

$$
\widehat{\Lambda_{j} * \Lambda_{j}}(\omega)=\left(\widehat{a^{j}}(\omega)\right)^{2} \widehat{\beta_{u_{2}}^{j}} \widehat{\Lambda_{u_{2}, j}}(\omega) .
$$

Going back to the time domain, we get

$$
\Lambda_{j} * \Lambda_{j}=\sum_{\ell \in \mathbb{Z}^{d}}\left(a^{j} * a^{j} * \beta_{u_{2}}^{j}\right)_{\ell} \Lambda_{u_{2}, j}\left(\cdot-M^{-j} \ell\right)=\sum_{\ell \in \mathbb{Z}^{d}} \gamma_{\ell}^{j} \Lambda_{u_{2}, j}\left(\cdot-M^{-j} \ell\right) .
$$

The vector $\gamma^{j}$ is the convolution of exponentially decaying vectors and then it decays exponentially. Using (4.18), (4.6) and the fact that $\Lambda_{j, u_{2}}$ is a cardinal function we get the proof.

Proposition 5.1 For any $e \in \mathcal{E}$, we define the vector $\alpha^{j, e}=\left(\alpha_{l}^{j, e}\right)_{l \in \mathbb{Z}^{d}}$ by

$$
\alpha_{l}^{j, e}=\left(\Lambda_{j+1}\left(\cdot-M^{-j-1} e\right), \Lambda_{j}\left(\cdot-M^{-j} l\right)\right)
$$

Then for any $e \in \mathcal{E}$

$$
\alpha_{l}^{j, e}=\left(c^{j} * \gamma^{j+1}\right)_{M l-e} \quad l \in \mathbb{Z}^{d},
$$

and

$$
p^{j, e}=\alpha^{j, e} * \gamma^{-j} .
$$

Proof Let us consider (5.11) and compute it in two different ways. First we apply the refinement equation (4.13) to $\Lambda_{j}\left(\cdot-M^{-j} l\right)$, and second we apply (5.5) to $\Lambda_{j+1}\left(\cdot-M^{-j-1} e\right)$. In both cases we use (5.7), the radiality of $\Lambda_{j}$ and $\Lambda_{u_{2}, j+1}$, and the symmetry of the vector $\beta_{u_{2}}^{j+1}$. In the first case we get

$$
\begin{aligned}
\alpha_{l}^{j, e} & =\left(\Lambda_{j+1}\left(\cdot-M^{-j-1} e\right), \Lambda_{j}\left(\cdot-M^{-j} l\right)\right)=\left(\Lambda_{j+1}\left(\cdot-M^{-j-1} e\right), \Lambda_{j}\left(M^{-j} l-\cdot\right)\right) \\
& =\left(\Lambda_{j+1}\left(\cdot-M^{-j-1} e\right), \sum_{k \in \mathbb{Z}^{d}} c_{k}^{j} \Lambda_{j+1}\left(M^{-j} l-\cdot-M^{-j-1} k\right)\right) \\
& =\sum_{k \in \mathbb{Z}^{d}} c_{k}^{j}\left(\Lambda_{j+1}\left(\cdot-M^{-j-1} e\right), \Lambda_{j+1}\left(M^{-j-1}(M l-k)-\cdot\right)\right) .
\end{aligned}
$$


The change of variable $M^{-j-1}(M l-k)-\cdot$ into $\cdot$, and (5.7) give

$$
\begin{aligned}
\alpha_{l}^{j, e} & =\sum_{k \in \mathbb{Z}^{d}} c_{k}^{j} \Lambda_{u_{2}, j+1}\left(M^{-j-1}(M l-e-k)\right) \\
& =\sum_{k \in \mathbb{Z}^{d}} c_{k}^{j} \gamma_{M l-e-k}^{j+1}=\left(c^{j} * \gamma^{j+1}\right)_{M l-e} .
\end{aligned}
$$

In the second case, we apply the decomposition equation (5.5). Since $\psi_{j}^{e} \perp \Lambda_{j}$, for any $l \in \mathbb{Z}^{d}$ and $e \in \mathcal{E}$ we get

$$
\alpha_{l}^{j, e}=\sum_{k \in \mathbb{Z}^{d}} p_{k}^{j, e}\left(\Lambda_{j}\left(\cdot-M^{-j} k\right), \Lambda_{j}\left(\cdot-M^{-j} l\right)\right) .
$$

The change of variable $\cdot-M^{-j} l$ into $\cdot$ gives

$$
\begin{aligned}
\alpha_{l}^{e} & =\sum_{k \in \mathbb{Z}^{d}} p_{k}^{j, e}\left(\Lambda_{j}\left(\cdot+M^{-j}(l-k)\right), \Lambda_{j}(\cdot)\right) \\
& =\sum_{k \in \mathbb{Z}^{d}} p_{k}^{j, e} \gamma_{l-k}^{j}=\left(p^{j, e} * \gamma^{j}\right)_{l} .
\end{aligned}
$$

Thus $\alpha^{j, e}=p^{j, e} * \gamma^{j}$, and since by (5.9) $\gamma^{j} * \gamma^{-j}=\delta$, we have $\alpha^{j, e} * \gamma^{-j}=p^{j, e}$.

Let us consider the decomposition filters $\left\{q^{j, e^{\prime}}\right\}_{e^{\prime} \in \mathcal{E}_{0}}$. For any $e^{\prime} \in \mathcal{E}_{0}$, the filter $q^{j, e^{\prime}}$ is defined by (5.6) in terms of the vectors $q^{j, e, e^{\prime}}, e \in \mathcal{E}$. The following Proposition shows that for all $e \in \mathcal{E}$ the vectors $\left\{q^{j, e, e^{\prime}}\right\}_{e^{\prime} \in \mathcal{E}_{0}}$ satisfy a linear system.

Proposition 5.2 For any $e \in \mathcal{E},\left\{q^{j, e, e^{\prime}}\right\}_{e^{\prime} \in \mathcal{E}_{0}}$ are such that linear system

$$
\sum_{\substack{k \in \mathbb{Z}^{d} \\ \widetilde{e}^{\prime} \in \mathcal{E}_{0}}} q_{k}^{j, e, \tilde{e}^{\prime}} a_{u_{2}, e^{\prime}-\widetilde{e}^{\prime}+M(l-k)}^{j+1}=a_{M l-e^{\prime}-e}^{j+1}, l \in \mathbb{Z}^{d}, e^{\prime} \in \mathcal{E}_{0} .
$$

Proof Let us define the vectors $\xi^{j, e, e^{\prime}}=\left\{\xi_{l}^{j, e, e^{\prime}}\right\}_{l \in \mathbb{Z}^{d}}, e \in \mathcal{E}, e^{\prime} \in \mathcal{E}_{0}$ as

$$
\xi_{l}^{j, e, e^{\prime}}:=\left(\Lambda_{j+1}\left(\cdot-M^{-j-1} e\right), \psi_{j}^{e^{\prime}}\left(\cdot-M^{-j} l\right)\right), e \in \mathcal{E}, e^{\prime} \in \mathcal{E}_{0},
$$

and compute $\xi_{l}^{j, e, e^{\prime}}$ in two different ways. First, we apply the refinement equation (5.4) to $\psi_{j}^{e^{\prime}}\left(\cdot-M^{-j} l\right)$, and second we apply (5.5) to $\Lambda_{j+1}\left(\cdot-M^{-j-1} e\right)$. In both cases we use (5.7), the radiality of $\Lambda_{j+1}$ and $u_{2}$, and the symmetry of the vector $\beta_{u_{2}}^{j+1}$. In the first case we get

$$
\begin{aligned}
\xi_{l}^{j, e, e^{\prime}} & =\left(\Lambda_{j+1}\left(\cdot-M^{-j-1} e\right), \psi_{j}^{e^{\prime}}\left(M^{-j} l-\cdot\right)\right) \\
& =\left(\Lambda_{j+1}\left(\cdot-M^{-j-1} e\right), \sum_{k \in \mathbb{Z}^{d}} d_{k}^{j} \Lambda_{j+1}\left(M^{-j} l-\cdot-M^{-j-1} e^{\prime}-M^{-j-1} k\right)\right) \\
& =\left(\Lambda_{j+1}\left(\cdot-M^{-j-1} e\right), \sum_{k \in \mathbb{Z}^{d}} d_{k}^{j} \Lambda_{j+1}\left(M^{-j-1}\left(M l-k-e^{\prime}\right)-\cdot\right)\right) .
\end{aligned}
$$


By the change of variable $\cdot=\cdot-M^{-j-1} e$ and using (5.1), and (5.8)

$$
\begin{aligned}
\xi_{l}^{j, e, e^{\prime}} & =\sum_{k \in \mathbb{Z}^{d}} d_{k}^{j}\left(\Lambda_{j+1}, \Lambda_{j+1}\left(M^{-j-1}\left(M l-k-e^{\prime}-e\right)-\cdot\right)\right) \\
& =\sum_{k \in \mathbb{Z}^{d}} d_{k}^{j} \gamma_{M l-e^{\prime}-e-k}^{j+1} \\
& =\left(d^{j} * \gamma^{j+1}\right)_{M l-e^{\prime}-e}=\left(\gamma^{j+1} * d^{j}\right)_{M l+e^{\prime}-e}=a_{M l-e^{\prime}-e}^{j+1} .
\end{aligned}
$$

Now we compute $\xi_{l}^{j, e, e^{\prime}}$ by using the decomposition equation (5.5), the definition (4.24) and the orthogonality between the spaces $V_{j}$ and $W_{j}$,

$$
\begin{aligned}
\xi_{l}^{j, e, e^{\prime}}= & \left(\Lambda_{j+1}\left(\cdot-M^{-j-1} e\right), \psi_{j}^{e^{e^{\prime}}}\left(M^{-j} l-\cdot\right)\right) \\
= & \sum_{\substack{k \in \mathbb{Z}^{d} \\
\widetilde{e}^{\prime} \in \mathcal{E}_{0}}} q_{k}^{j, e, \tilde{e}^{\prime}}\left(\psi_{j}^{\tilde{e}^{\prime}}\left(\cdot-2^{-j} k\right), \psi_{j}^{e^{\prime}}\left(\cdot-M^{-j} l\right)\right) \\
= & \sum_{\substack{k \in \mathbb{Z}^{d} \\
\widetilde{e}^{\prime} \in \mathcal{E}_{0}}} q_{k}^{j, e, \tilde{e}^{\prime}}\left(\psi_{j}\left(\cdot-M^{-j-1} \widetilde{e}^{\prime}-M^{-j} k\right), \psi_{j}\left(M^{-j} l-\cdot-M^{-j-1} e^{\prime}\right)\right) \\
= & \sum_{\substack{k \in \mathbb{Z}^{d} \\
\widetilde{e} \in E^{\prime}}} q_{k}^{j, e, \tilde{e}^{\prime}}\left(\psi_{j}\left(\cdot-M^{-j-1}\left(\widetilde{e}^{\prime}+M k\right)\right), \psi_{j}\left(-M^{-j-1}\left(e^{\prime}+M l\right)-\cdot\right)\right)
\end{aligned}
$$

By applying (5.3) and changing $M^{-j-1}\left(e^{\prime}+M l\right)-\cdot$ in $\cdot$, we get

$$
\begin{aligned}
\xi_{l}^{j, e, e^{\prime}} & =\sum_{\substack{k \in \mathbb{Z}^{d} \\
\widetilde{e}^{\prime} \in E^{\prime}}} q_{k}^{j, e, \tilde{e}^{\prime}}\left(\psi_{j}\left(M^{-j-1}\left(\widetilde{e}^{\prime}+e^{\prime}-M k+M l\right)-\cdot\right), \psi_{j}(\cdot)\right) \\
& =\sum_{\substack{k \in \mathbb{Z}^{d} \\
\widetilde{e}^{\prime} \in \mathcal{E}_{0}}} q_{k}^{j, e, \tilde{e}^{\prime}} \sum_{i, s \in \mathbb{Z}^{d}} d_{i}^{j} d_{s}^{j}\left(\Lambda_{j+1}\left(M^{-j-1}\left(-\widetilde{e}^{\prime}-e^{\prime}-M k+M l-i-s\right)-\cdot\right), \Lambda_{j+1}(\cdot)\right) \\
& =\sum_{\substack{k \in \mathbb{Z}^{d} \\
\widetilde{e}^{\prime} \in \mathcal{E}_{0}}} q_{k}^{j, e, \tilde{e}^{\prime}} \sum_{i, s \in \mathbb{Z}^{d}} d_{i}^{j} d_{s}^{j} \gamma_{\widetilde{e}^{\prime}+e^{\prime}-M k+M l-i-s}^{j+1} \cdot
\end{aligned}
$$

By changing $s$ in $i-h$, we obtain

$$
\begin{aligned}
\xi_{l}^{j, e, e^{\prime}} & =\sum_{\substack{k \in \mathbb{Z}^{d} \\
\tilde{e}^{\prime} \in \mathcal{E}_{0}}} q_{k}^{j, e, \tilde{e}^{\prime}} \sum_{i, h \in \mathbb{Z}^{d}} d_{i}^{j} d_{i-h}^{j} \gamma_{-\widetilde{e}^{\prime}+e^{\prime}+M l-M k-h}^{j+1} \\
= & \sum_{\substack{k \in \mathbb{Z}^{d} \\
\widetilde{e}^{\prime} \in \mathcal{E}_{0}}} q_{k}^{j, e, \tilde{e}^{\prime}} \sum_{h \in \mathbb{Z}^{d}}\left(d^{j} * d^{j}\right)_{h} \gamma_{-\widetilde{e}^{\prime}+e^{\prime}+M l-M k-h}^{j+1} \\
= & \sum_{\substack{k \in \mathbb{Z}^{d} \\
\widetilde{e}^{\prime} \in \mathcal{E}_{0}}} q_{k}^{j, e, \tilde{e}^{\prime}}\left(d^{j} * d^{j} * \gamma^{j+1}\right)_{-\widetilde{e}^{\prime}+e^{\prime}+M(l-k) .}
\end{aligned}
$$


Now remembering that $a_{u_{2}}^{j+1} * \beta_{u_{2}}^{j+1}=\delta($ see $(4.18)), a^{j+1} * \beta^{j+1}=\delta(\operatorname{see}(4.6))$, and using (5.1), (5.8) we have

$$
\begin{aligned}
d^{j} * d^{j} * \gamma^{j+1} & =a_{u_{2}}^{j+1} * \beta^{j+1} * a_{u_{2}}^{j+1} * \beta^{j+1} * a^{j+1} * a^{j+1} * \beta_{u_{2}}^{j+1} \\
& =a_{u_{2}}^{j+1} * \beta^{j+1} * a_{u_{2}}^{j+1} * a^{j+1} \beta_{u_{2}}^{j+1} \\
& =a_{u_{2}}^{j+1} * \beta^{j+1} * a_{u_{2}}^{j+1} * \beta_{u_{2}}^{j+1} * a^{j+1} \\
& =a_{u_{2}}^{j+1} * \beta^{j+1} * a^{j+1}=a_{u_{2}}^{j+1},
\end{aligned}
$$

so

$$
\xi_{l}^{j, e, e^{\prime}}=\sum_{\substack{k \in \mathbb{Z}^{d} \\ \widetilde{e}^{\prime} \in \mathcal{E}_{0}}} q_{k}^{j, e, \tilde{e}^{\prime}} a_{u_{2},-\widetilde{e}^{\prime}+e^{\prime}+M(l-k)}^{j+1} .
$$

Comparing the expressions we get the proof.

\section{The decomposition and reconstruction of a signal}

A signal $\sigma_{n+1} \in V_{n+1}$ admits a unique representation in $V_{n}+W_{n}$, that is $\sigma_{n+1}=\sigma_{n}+\sigma_{n}^{w}$, where $\sigma_{n}=\sum_{\ell \in \mathbb{Z}^{d}} \lambda_{\ell}^{n} \Lambda_{n}\left(x-M^{-n} \ell\right)$ is the coarse approximation of $\sigma_{n+1}$ in $V_{n}$ and $\sigma_{n}^{w}=\sum_{e \in \mathcal{E}_{0}} \sum_{\ell \in \mathbb{Z}^{d}} \mu_{k}^{n, e} \psi_{n}^{e}\left(x-M^{-n} \ell\right)$ represents the detail function of $\sigma_{n+1}$ in $W_{n}$. The vectors $\lambda^{n}$ and $\mu^{n, e}, e \in \mathcal{E}_{0}$ are respectively called approximation coefficients and detail coefficients, and in a decomposition/reconstruction process they represent $\sigma_{n}$ and $\sigma_{n}^{w}$. The nonstationary wavelet decomposition/reconstruction of $\sigma_{n+1} \in V_{n+1}$ involves at each step convolution between the approximation coefficients and the decomposition filters $p^{n}$ and $q^{n, e^{\prime}}\left(e^{\prime} \in \mathcal{E}_{0},\right)$ and downsampling (with respect to the matrix $M$ ), upsampling (with respect to the matrix $M$ ), and convolutions between the approximation and detail coefficients with the reconstruction filters $c^{n}$ and $d^{n}$.

\subsection{Computation via convolution products}

Performing a certain number $s$ of decomposition we get the following situation

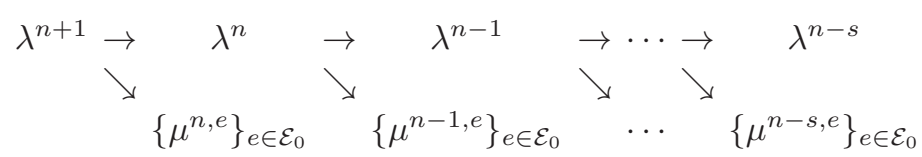

where the arrows indicate convolution followed by a downsampling. For the reconstruction we can go backwards to obtain $\lambda^{n+1}$

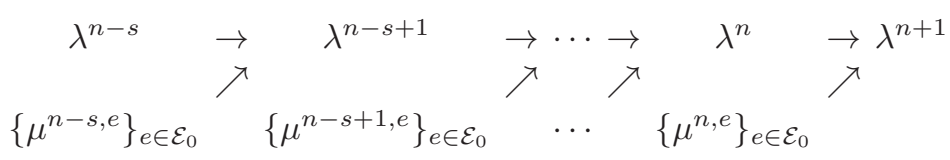





Fig. 6.1 The decomposition filters when $j=0, M=2 I, m=2, \kappa_{1}=3, \kappa_{2}=4$.

where the upsampling is required before convolutions. At each level the filters are different and the number of filters is $|\operatorname{det} M|$ which is strictly connected with the complexity. $M$ gives also the cosets and then the directions along which a signal can be analysed. The construction of an efficient multiresolution algorithm in the general case is a non-trivial task and it is beyond the purposes of this paper.

All the implied summations are infinite summations. However since the implied vectors are exponentially decaying, we can approximate the exact solution, up to an appropriate precision, by a truncation of it. Unfortunately we did not get any bound of the rate of decay, or of the absolute value of the terms of the filters. These values depend non linearly on the values of the $\kappa_{\ell}$ 's, and a general bound is not easy to be obtained. So we suggest, when needing to use these vectors, to use an experimental decay to decide the exact number of components to take into account. This is what we did to compute the decomposition filters presented in Figures 6.1 and 6.2 for $m=2, d=2$ and different values of $\kappa_{1}$ and $\kappa_{2}$ when $j=0$ and $M=2 I$.

Various experiments have shown that $21 \times 21$ a sufficient filter size to get good results since the absolute values of the truncated components of the various filter are less than $1.1 \mathrm{e}-11$, and this value decreases fast to the roundoff unit when the parameters $\kappa_{\ell}$ increase. The important point is to do a truncation that provides numerically supported filters and this allows to get in general results as good as those produced by compactly supported filters (see e.g. [3]). 

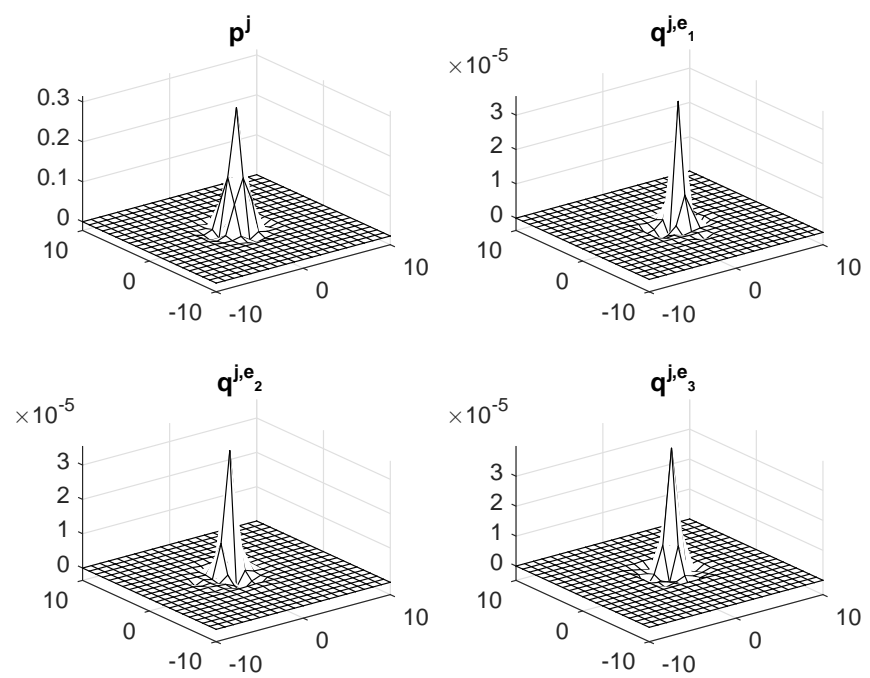

Fig. 6.2 The decomposition filters when $j=0, M=2 I, m=2, \kappa_{1}=3, \kappa_{2}=21$.

\subsection{Examples of signal decomposition}

As test images, let us consider the well-known phantom image of size $512 \times 512$ and the image obtained sampling the function (6.1) at $256 \times 256$ points (Fig. $6.3)$

$$
f(x, y)=f_{1}(x) f_{2}(y)
$$

where

$$
f_{1}(x)=\left\{\begin{array}{l}
0, x \in[0,1] \cup[2,3] \cup[4,5] \\
1, x \in(1,2) \\
2, x \in(3,4),
\end{array} \quad f_{2}(x)= \begin{cases}\sin (x), & x \in[0, \pi] \\
2|\sin (x)|, & x \in(\pi, 2 \pi) \\
3 \sin (x) & x \in[2 \pi, 3 \pi]\end{cases}\right.
$$

Let us perform a one-level decomposition. We fix $m=2$, and $\kappa_{1}=3$. An extensive experimentation, has shown that when $\kappa_{2}$ increases, the details become more and more better defined. Here we show only the results obtained for $\kappa_{2}=4,21$ (see Figs. 6.5, 6.6, 6.9, and 6.10) and a comparison with the non separable polyharmonic B-spline wavelet decomposition [2] (Figs. 6.7, 6.11) and the classical tensor product wavelet decomposition obtained with biorthogonal wavelets (bior3.3)(Figs. 6.8, 6.12)

The analysis of the result is quite clear: if we are only interested by file compression, the up-left resulting image, the tensorial product wavelet decomposition (figure $6.8,6.12$ ) is the more interesting one. However if we are interested in edge detection, as it is more and more the case, Fig. 6.6 is clearly much better than Fig. 6.8. So this depends on what we are interested in, and the GWM functions are of great interest in edge detection. This shows that the non-separable and non-stationary wavelet decomposition presented in the 
paper is potentially better and more effective thanks to the parameters for some applications.
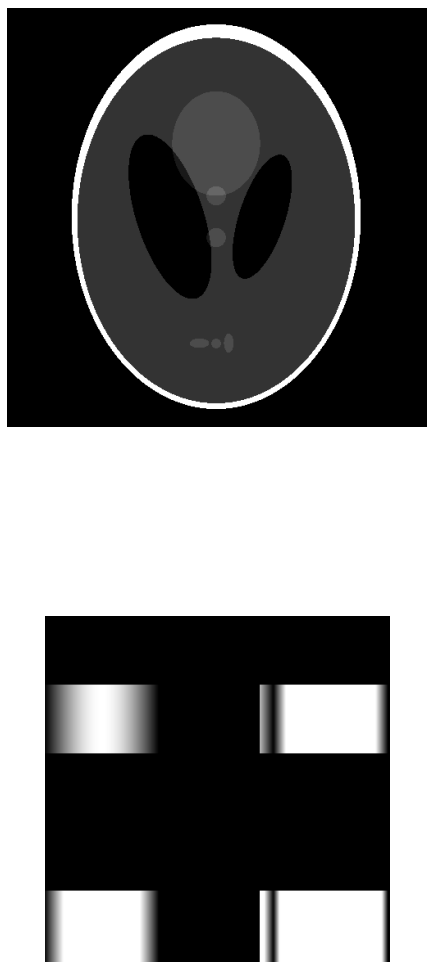

Fig. 6.3 Top: The Phantom test image. Bottom: the test image obtained from (6.1).

\section{Conclusion}

The GWM functions are useful to modeling, in any number of variables, functions or processes of various shape properties, such us preserving peaks at the data points, or on the opposite being quite flat. They are important generalizations of polyharmonic splines and Whittle Matérn kernels and indeed they are between these two well-known families. Their interest is the possibility for the user to have tension parameters. The existence of the parameters $\kappa_{\ell}$ implies a non stationary multi-resolution analysis and a non-stationary convergent 


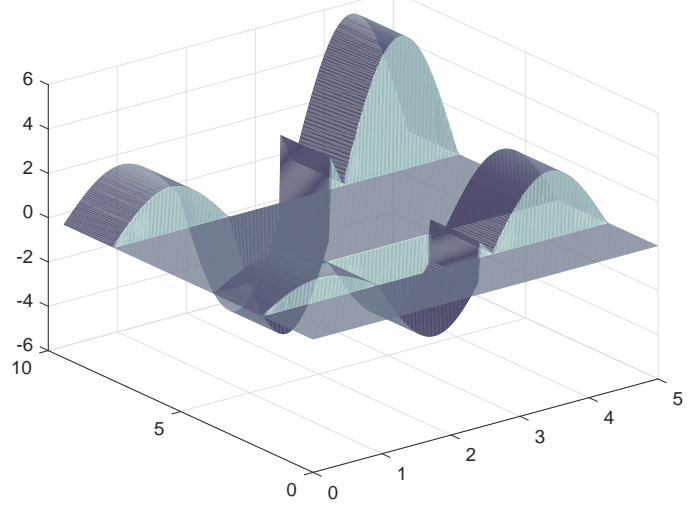

Fig. 6.4 The function (6.1).

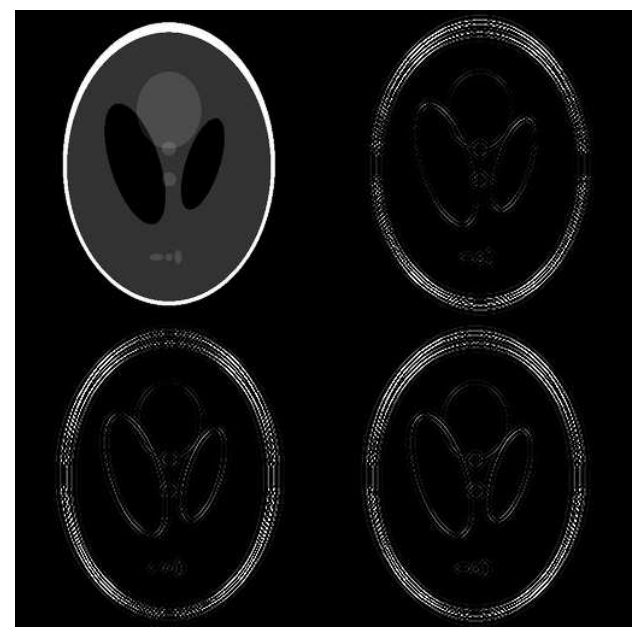

Fig. 6.5 $\kappa_{2}=4$. Top left: the approximation coefficients. Top right: the horizontal details. Bottom left: the vertical details. Bottom right: the diagonal details.

subdivision scheme. We gave all technical and theoretical tools to manipulate these functions, emphasing those necessary to handle a specific multiresolution analysis. The main theoretical tool for that is the Fourier transform, which is directly due to the definition of the GWM kernels which is straightfully converted in a quite simple function in the Fourier domain; the main technical tool for implementing the NSMRA is the truncated convolution product.

We have shown with some examples the crucial role played by the parameters $\left\{\kappa_{\ell}\right\}_{\ell=1}^{m}$ in various situations. The signal decomposition examples 


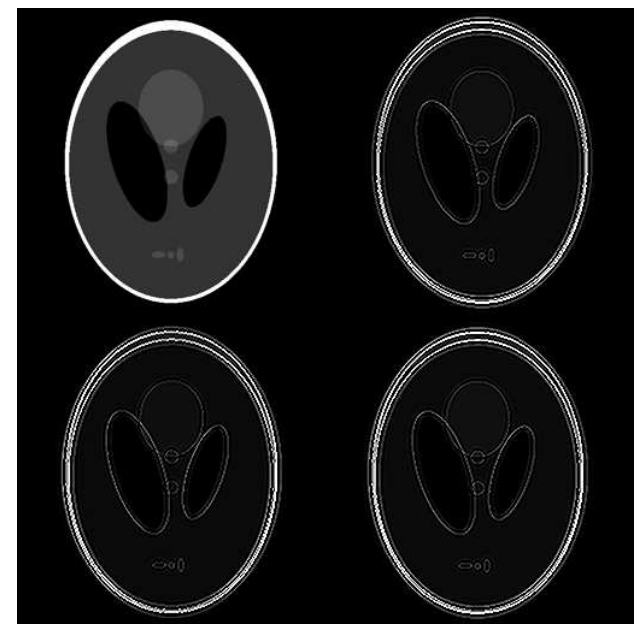

Fig. 6.6 $\kappa_{2}=21$. Top left: the approximation coefficients. Top right: the horizontal details. Bottom left: the vertical details. Bottom right: the diagonal details.

highlight the details and the discontinuities in the images, which gives various types of concrete use of these functions, in particular for edge detection.

Acknowledgements We would like to thank the anonymous reviewers for their valuable remarks and suggestions.

\section{References}

1. H. Akima. A new method of interpolation and smooth curve fitting based on local procedure. J. Assoc. Comput. Mach., 17(4):589-602, 1970.

2. Barbara Bacchelli, Mira Bozzini, Christophe Rabut, and Maria Varas. Decomposition and reconstruction of multidimensional signals using polyharmonic pre-wavelets. Applied and Computational Harmonic Analysis, 18(3):282-299, 2005.

3. Barbara Bacchelli, Mira Bozzini, and Milvia Rossini. On the errors of multidimensional MRA based on non-separable scaling functions. Int. J. Wavelets Multiresolut. Inf. Process., 4(3):475-488, 2006.

4. A. Bouhamidi. Hilbertian approach for univariate spline with tension. Approx. Theory Appl., 17(4):36-57, 2001.

5. Mira Bozzini, Milvia Rossini, and Robert Schaback. Generalized Whittle-Matérn and polyharmonic kernels. Adv. Comput. Math., 39(1):129-141, 2013.

6. M. D. Buhmann. Radial basis functions: theory and implementations, volume 12 of Cambridge Monographs on Applied and Computational Mathematics. Cambridge University Press, Cambridge, 2003.

7. C. K. Chui, J. Stöckler, and J. D. Ward. Analytic wavelets generated by radial functions. Adv. Comput. Math., 5(1):95-123, 1996.

8. Carl de Boor, Ronald A. DeVore, and Amos Ron. On the construction of multivariate (pre)wavelets. Constr. Approx., 9(2-3):123-166, 1993. 


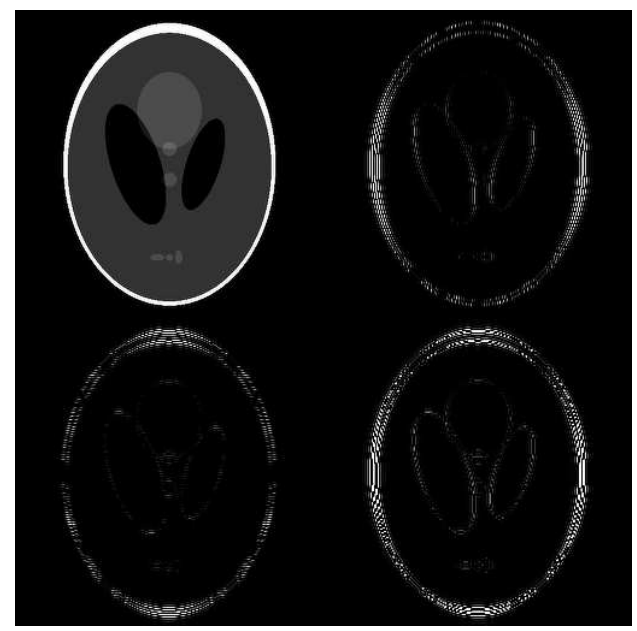

Fig. 6.7 Polyharmonic B-spline wavelet decomposition. Top left: the approximation coefficients. Top right: the horizontal details. Bottom left: the vertical details. Bottom right: the diagonal details.

9. Jean Duchon. Interpolation des fonctions de deux variables suivant le principe de la flexion des plaques minces. Rev. Francaise Automat. Informat. Recherche Opérationnelle Sér. RAIRO Analyse Numérique, 10(R-3):5-12, 1976.

10. Gregory E. Fasshauer. Green's functions: taking another look at kernel approximation, radial basis functions, and splines. In Approximation theory XIII: San Antonio 2010, volume 13 of Springer Proc. Math., pages 37-63. Springer, New York, 2012.

11. Armin Iske. Multiresolution methods in scattered data modelling, volume 37 of Lecture Notes in Computational Science and Engineering. Springer-Verlag, Berlin, 2004.

12. Ildar Khalidov, Michael Unser, and John Paul Ward. Operator-like wavelet bases of $L_{2}\left(\mathbb{R}^{d}\right)$. J. Fourier Anal. Appl., 19(6):1294-1322, 2013.

13. Per Erik Koch and Tom Lyche. Exponential $B$-splines in tension. In Approximation theory VI, Vol. II (College Station, TX, 1989), pages 361-364. Academic Press, Boston, MA, 1989.

14. W. R. Madych. Some elementary properties of multiresolution analyses of $L^{2}\left(\mathbf{R}^{n}\right)$. In Wavelets, volume 2 of Wavelet Anal. Appl., pages 259-294. Academic Press, Boston, MA, 1992 .

15. W. R. Madych. Spline type summability for multivariate sampling. In Analysis of divergence (Orono, ME, 1997), Appl. Numer. Harmon. Anal., pages 475-512. Birkhäuser Boston, Boston, MA, 1999.

16. C. Rabut and M. Rossini. Polyharmonic multiresolution analysis: An overview and some new results. Numerical Algorithms, 48(1-3):135-160, 2008.

17. L.L. Schumaker. Spline Functions: Basic Theory. Wiley-Interscience, 1981.

18. Joachim Stöckler. Non-stationary wavelets. In Multivariate approximation: from CAGD to wavelets (Santiago, 1992), volume 3 of Ser. Approx. Decompos., pages 307-320. World Sci. Publ., River Edge, NJ, 1993.

19. M. Unser and T. Blu. Cardinal exponential splines: Part I - Theory and filtering algorithms. IEEE Trans. Signal Proc. Networks, 53:1425-1438, 2005.

20. Dimitri Van De Ville, Thierry Blu, and Michael Unser. Isotropic polyharmonic B-splines: scaling functions and wavelets. IEEE Trans. Image Process., 14(11):1798-1813, 2005. 


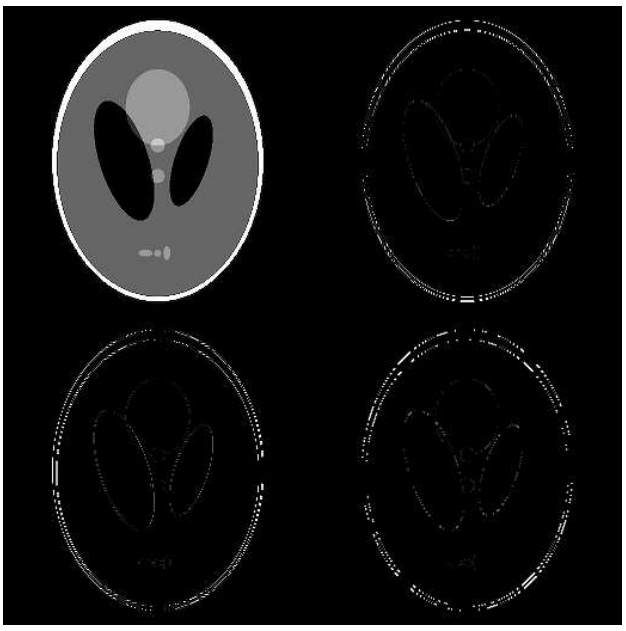

Fig. 6.8 Tensor-product wavelet decomposition: bior3.3. Top left: the approximation coefficients. Top right: the horizontal details. Bottom left: the vertical details. Bottom right: the diagonal details.



Fig. 6.9 $\kappa_{2}=4$. Top left: the approximation coefficients. Top right: the horizontal details Bottom left: the vertical details. Bottom right: the diagonal details. 




Fig. 6.10 $\kappa_{2}=21$. Top left: the approximation coefficients. Top right: the horizontal details. Bottom left: the vertical details. Bottom right: the diagonal details.



Fig. 6.11 Polyharmonic B-spline wavelet decomposition. Top left: the approximation coefficients. Top right: the horizontal details. Bottom left: the vertical details. Bottom right: the diagonal details. 


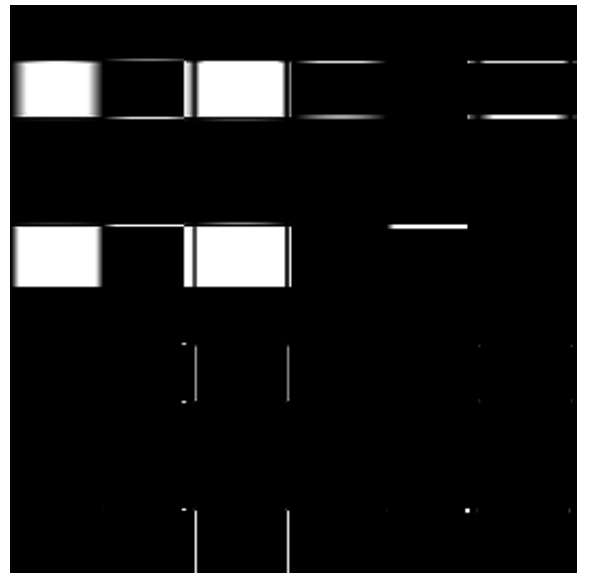

Fig. 6.12 Tensor-product wavelet decomposition: bior3.3. Top left: the approximation coefficients. Top right: the horizontal details. Bottom left: the vertical details. Bottom right: the diagonal details. 